\title{
Hidrogeoquímica de salinas Zapotitlán y los lagos-cráter Alchichica y Atexcac, Puebla
}

\author{
Hydrogeochemistry of salinas Zapotitlan and the crater-lakes \\ Alchichica and Atexcac, Puebla
Oscar Raúl Mancilla Villa ${ }^{1 *}$, Ana Laura Bautista Olivas ${ }^{1}$, Héctor Manuel Ortega Escobar ${ }^{1}$, Edgar Iván Sánchez Bernal ${ }^{2}$, Álvaro Can Chulim ${ }^{3}$, Rubén Darío Guevara Gutiérrez ${ }^{4}$ e Ygor Manuel Ortega Mikolaev ${ }^{2}$

\section{RESUMEN}

\begin{abstract}
En este trabajo se procedió a la evaporación experimental de salmueras procedentes de dos lagos-cráter salinos, Alchichica y Atexcac, de la cuenca del Oriental, Puebla, y de un manantial salino, Salinas Chiquitas, de Zapotitlán, Puebla, para documentar de este modo las pautas de evolución hasta las etapas de mayor concentración de las soluciones. Además se consideraron 214 aguas de baja concentración que transitan las cuencas de Puebla, Tlaxcala y Veracruz, para conocer y comparar la composición hidrogeoquímica de las soluciones analizadas procedentes de diferentes zonas geográficas de México. El experimento de evaporación se llevó a cabo en condiciones isotermas, a $50^{\circ} \mathrm{C}$ y hasta total desecación del sistema, durante el cual se recogieron muestras de salmueras en las que se analizaron sus contenidos de aniones y cationes, así como en las aguas de baja concentración. Las tres salmueras son similares en su composición química, presentan soluciones de tipo $\mathrm{Na}-\mathrm{Cl}$, con importantes variaciones en el contenido de $\mathrm{SiO}_{2}$ y de $\mathrm{HCO}_{3}$; por su parte las aguas de baja concentración son soluciones de tipo $\mathrm{HCO}_{3}$-Ca. Las vías evolutivas geoquímicas que presentan las aguas de baja concentración son en su mayoría, en primer lugar carbonatada y en segundo lugar cálcica. Por su parte las salmueras de los lagos-cráter y del manantial de Zapotitlán presentan la vía sulfatada directa.
\end{abstract}

Palabras clave: evaporación de salmueras, geoquímica, carbonatada, cálcica, sulfatada.

\begin{abstract}
In this study we carried on the experimental evaporation of brines from two saline crater-lakes: Alchichica and Atexcac, in the Basin of the Oriental, Puebla, and from a saline spring, Salinas Chiquitas, Zapotitlán, Puebla, thus documenting patterns of evolution to higher stages of concentration of solutions. In addition 214 low concentration waters were considered which flow in the basins of Puebla, Tlaxcala and Veracruz, to know and compare the hydrogeochemical composition of the solutions analyzed from different geographical areas of Mexico. The experiment of evaporation was carried out under isothermal conditions at $50{ }^{\circ} \mathrm{C}$ until complete drying of the system, during this process brine samples were collected in which their anions and cations contents were analyzed, as well as in low water concentration. The three brines are similar in chemical characteristics, they present $\mathrm{Na-Cl}$ type solutions, with significant variations in the content of $\mathrm{SiO}_{2}$ and $\mathrm{HCO}_{3}$; meanwhile low concentration waters are $\mathrm{HCO}_{3}$-Ca type solutions. The geochemical developmental pathways that low concentration waters have are mostly first carbonated and second calcium. Meanwhile the brines of the crater-lakes and the saline spring of Zapotitlán present the direct sulfated route.
\end{abstract}

Key words: evaporation of brines, geochemistry, carbonated, calcium, sulfated.

\section{Introducción}

En la zona de Zapotitlán, Puebla, se tienen un sinnúmero de manantiales salinos en los cuales por medio de la evaporación se obtienen cantidades de sal que los pobladores de esta zona comercializan. Son aguas freáticas salinas que circulan fallas, debido a los plegamientos que ha sufrido la formación

1 Departamento de Producción Agrícola del Centro Universitario de la Costa Sur, Universidad de Guadalajara; Av. Independencia Nacional \# 151 C.P. 48900 , Autlán de Navarro, Jalisco, México.

2 Instituto de Ecología. Universidad del Mar Campus Puerto Ángel; Ciudad Universitaria, Puerto Ángel, Pochutla, Oaxaca, México. C.P. 70902.

3 Universidad Autónoma de Nayarit, Ciudad de la Cultura Amado Nervo. Tepic, Nayarit. México. C.P. 63155.

4 Universidad de Guadalajara, Centro Universitario de la Costa Sur, Departamento de Ecología y Recursos Naturales, Av. Independencia Nacional 151, Autlán de Navarro, Jal. 48900.

* Autor por correspondencia: oscar.mancilla@ cucsur.udg.mx

Fecha de Recepción: 4 Enero, 2013.

Fecha de Aceptación: 21 Noviembre, 2013. 
Zapotitlán en distintos periodos geológicos. Las aguas salinas en sus recorridos hacia los diferentes alumbramientos atraviesan diferentes espesores de evaporitas sepultadas de origen marino y volcánico (Can, 2011).

La conformación general de la fauna, considerada junto con la litología de las rocas, sugiere que la región de Zapotitlán-San Juan Raya, a fines del Neocomiano y durante el Aptiano, fue un área cercana a la costa, cubierta por aguas marinas poco profundas. La presencia de abundantes corales y rudistas confirma esta conclusión, y sugiere que las aguas tuvieron una temperatura templada (ReyesCortés, 1979).

Los lagos-cráter o Maars se originaron debido a explosiones volcánicas que ocasionaron fallas y rupturas en los espesores saturados de alumbramientos de aguas subterráneas. Con el tiempo los lagos-cráter de la cuenca del Oriental como son: Alchichica, La Preciosa, Quechulac, Aljojuca, Tecuitlapa y Atexcac se llenaron de agua. Las aguas de los lagos-cráter de Alchichica y Atexcac son salinas (Can, 2011; Arredondo et al., 1983; Gasca, 1982, Arredondo, 2002).

Arredondo et al. (1983) mencionan que en cuanto a la geología regional la planicie de la cuenca del Oriental alcanzó su elevación debido al plegamiento de rocas de origen marino del mesozoico, a la acumulación de rocas y derrames volcánicos y a la enorme cantidad de sedimentos piroclásticos, que en última instancia han dado la configuración actual a la cuenca. Las formas fisiográficas volcánicas se han producido desde principios del Cenozoico hasta el Cuaternario y de esta manera se configura la clásica fisiografía de Malpaís (Reyes, 1979; Alcocer et al., 2005).

La concentración de los cationes en los lagoscráter, sigue el orden de: $\mathrm{Na}^{+}>\mathrm{Mg}^{2+}>\mathrm{Ca}^{2+}$ y respecto de los aniones, para los lagos Alchichica, Atexcac y La Preciosa, por lo general siguen el orden de: $\mathrm{Cl}^{-}>\mathrm{HCO}_{3}^{-}>\mathrm{CO}^{2-}{ }_{3}>\mathrm{SO}_{-4}{ }_{-4}$ y para los lagos Quechulac, Aljojuca y Tecuitlapa el orden es: $\mathrm{HCO}_{3}^{-}>\mathrm{Cl}^{-}>\mathrm{CO}^{2-}{ }_{3}>\mathrm{SO}^{2-}{ }_{4}(\mathrm{Can}, 2011)$. Las comparaciones demuestran que los iones más abundantes de los lagos-cráter son $\mathrm{Na}^{+} \mathrm{Mg}^{2+} \mathrm{Cl}^{-}$y $\mathrm{HCO}_{3}^{-}$y esta composición química se asemeja a la del agua de mar, donde los iones más abundantes son $\mathrm{Na}^{+} \mathrm{Mg}^{2+}, \mathrm{Cl}^{-}$y $\mathrm{SO}^{2-}{ }_{4}$ (Can, 2011).

En los estudios de las vías evolutivas geoquímicas que adquieren las aguas salinas durante un proceso de evaporación, se estudió el Manantial de Las Salinas Chiquitas de Zapotitlán, Puebla, y las aguas salinas de los lagos-cráter de Alchichica y Atexcac. Además se analizaron geoquímicamente 214 aguas superficiales de baja concentración iónica que transitan en los estados de Puebla, Tlaxcala y Veracruz, con la finalidad de conocer la composición iónica de las soluciones salinas de diferentes regiones de México y comparar los resultados con otras investigaciones en el tema, en diferentes zonas del mundo.

\section{Metodología}

Los lagos se encuentran localizados en la Cuenca del Oriental, situada entre los estados de Puebla, Tlaxcala y Veracruz en las coordenadas $97^{\circ} 09^{\prime}$ y $98^{\circ} 03^{\prime}$ de longitud oeste y a los $18^{\circ} 48^{\prime}$ y $19^{\circ} 43^{\prime}$ de latitud norte (Gasca, 1982). Los dos grandes llanos que forman parte de esta cuenca son de origen lacustre, llamados Llanos de San Andrés y Llanos de San Juan, que se encuentran al noroeste de la meseta poblana entre los poblados de San Salvador el Seco y Perote. En los Llanos de San Andrés se encuentran los lagos Aljojuca y Tecuitlapa y en los Llanos de San Juan, los lagos Atexcac, La Preciosa, Quechulac y Alchichica. En la Tabla 1 se presentan las características morfométricas generales de los lagos-cráter del estado de Puebla. El valle de Zapotitlán está situado en la zona sureste de Puebla y pertenece a la región de la Mixteca Baja. También es una prolongación hacia el oeste del valle de Tehuacán, región mejor conocida desde los trabajos de MacNeish et al. (1972).

Tabla 1. Características morfométricas generales de los lagos-cráter de Puebla.

\begin{tabular}{lcccccc}
\hline \multicolumn{1}{c}{ Concepto } & Alchichica & La Preciosa & Quechulac & Aljojuca & Atexcac & Tecuitlapa \\
\hline Área superficial $\left(\mathrm{km}^{2}\right)$ & 1,81 & 0,78 & 0,50 & 0,44 & 0,29 & 0,26 \\
Volumen $\left(\mathrm{m}^{3} \times 10^{6}\right)$ & 69,90 & 16,20 & 10,90 & 11,60 & 6,10 & 0,35 \\
Profundidad Máxima $(\mathrm{m})$ & 64,60 & 45,50 & 4,00 & 50,60 & 39,10 & 2,50 \\
\hline
\end{tabular}

Datos tomados de Arredondo et al. (1983), 
El Cretácico Inferior de la región Zapotitlán Salinas de donde se tomaron las muestras de agua salina para el estudio de las vías evolutivas, durante un proceso de evaporación continua, comprende las formaciones Zapotitlán y San Juan Raya (Can, 2011). La formación Zapotitlán pertenece al Barremiano y se conforma de una serie de lutitas, calizas con paquiodontes y conglomerados.

Para los estudios de las vías evolutivas geoquímicas que siguen las aguas salinas de los lagos-cráter Alchichica y Atexcac, además del manantial de Salinas Chiquitas de Zapotitlán, Puebla, se colectaron $2 \mathrm{~L}$ de agua salina de cada sitio. El agua se virtió en un vaso de precipitado, para cada sitio, para colocarlo en una parrilla de secado e iniciar la evaporación.

Después de haberse iniciado el proceso de evaporación, enseguida cada $100 \mathrm{ml}$ evaporados se tomó una alícuota de agua para hacer los análisis químicos correspondientes. En total se tomaron 20 muestras de agua de cada sitio. El proceso de evaporación en el laboratorio se llevó a cabo en condiciones isotérmicas $\left(50{ }^{\circ} \mathrm{C}\right)$. Este valor de temperatura es muy próximo a la temperatura a la que se lleva a cabo la evaporación en condiciones naturales en las salinas Chiquitas en Zapotitlán, Puebla. Las sales hipotéticas se calcularon de acuerdo con lo reportado por Mendoza (2009). En la Tabla 2 se presentan las determinaciones fisicoquímicas analíticas que se realizaron a cada muestra de agua de las aguas de Alchichica, Atexcac y Zapotitlán Salinas, Puebla, y las aguas de tres muestreos realizados en 2009, 2010 y 2011.

Para determinar qué vía evolutiva geoquímica seguirá un agua en particular enseguida se presentan las siguientes relaciones (Risacher, 1996a; Risacher, 1996b): $[\mathrm{Alc}]>[\mathrm{Ca}]$ y $[\mathrm{Alc}]>[\mathrm{Ca}]+[\mathrm{Mg}]$, vía carbonatada. $[\mathrm{Alc}]>[\mathrm{Ca}]$ y $[\mathrm{Alc}]<[\mathrm{Ca}]+[\mathrm{Mg}]$, vía sulfatada alcalina directa. [Alc] $<[\mathrm{Ca}]$ y $[\mathrm{Alc}]$ $+\left[\mathrm{SO}_{4}\right]>[\mathrm{Ca}]$, vía sulfatada neutra. $[\mathrm{Alc}]<[\mathrm{Ca}]$ $\mathrm{y}[\mathrm{Alc}]+\left[\mathrm{SO}_{4}\right]<[\mathrm{Ca}]$, vía evolutiva geoquímica cálcica, Alc $=$ Alcalinidad $\left(\mathrm{Alc}=\mathrm{CO}_{3}+\mathrm{HCO}_{3}\right)$, (Risacher et al., 1999; Hardie y Eugster, 1970).

\section{Resultados y discusión}

Durante el proceso de escurrimiento de corrientes fluviales a través de cuencas determinadas, la composición química de las aguas tiene variaciones, además de que en ciertos procesos de evaporación las soluciones acuosas que circulan en los ríos y manantiales tienden a adquirir composiciones químicas diversas, sin embargo, a pesar de que las composiciones químicas sean variadas, es posible predeterminar cuáles serán las vías geoquímicas de evolución que estas aguas irán adquiriendo en un proceso de aumento de la concentración total electrolítica (Hardie y Eugster, 1970; Eugster y Hardie, 1978; Risacher y Alonso, 1996; ; Risacher y Alonso, 1996 : Risacher et al., 1999: Risacher y Fritz, 1991).

En un sistema trilineal de composiciones iónicas, tanto de cationes como de aniones, se presentan en forma de diagrama, todos los valores iónicos $\left(\mathrm{mmol}_{\mathrm{c}} \mathrm{L}^{-1}\right)$ de todas las muestras de agua de tres muestreos realizados en los estados de Tlaxcala, Puebla y Veracruz en 2009, 2010 y 2011 (Figura 1). Se puede observar que la gran mayoría de las muestras de agua son de composición bicarbonatada. Cuando se cuenta con una considerable cantidad de composiciones químicas de muestras de agua que han sido colectadas, como es el caso de esta investigación,

Tabla 2. Determinaciones fisicoquímicas para aguas superficiales de los estados de Tlaxcala, Puebla y Veracruz y las aguas salinas de Alchichica, Atexcac y Zapotitlán salinas.

\begin{tabular}{rlll}
\hline \multicolumn{1}{c}{ Determinación } & & \multicolumn{1}{c}{ Método } \\
\hline 1 & $\mathrm{pH}$ & Potenciométrico & Refencia \\
2 & Conductividad Eléctrica & Conductimetría & Eaton et al., 1995 \\
3 & Residuo seco E. & Gravimetría & Richards, 1990 \\
4 & Sodio y potasio & Flamometría & Eaton et al., 1995 \\
5 & Calcio y magnesio & Volumétrico & APHA, 1995 3500- $\mathrm{Na}^{+} \mathrm{y} \mathrm{K}^{+}, \mathrm{D}$ \\
6 & Carbonatos & Volumétrico & APHA, 1995 3500 Ca ${ }^{2+}, \mathrm{D}$ \\
7 & Bicarbonatos & Volumétricos & APHA, 1995 2320 B \\
8 & Cloruros & Titulación & APHA, 1995 2320 B \\
9 & Sulfatos & Turbidimetría & APHA, 1995 4500-C-IB \\
10 & Silicio & Turbidimetría & APHA, 1995 4500-SO ${ }_{4}^{2-} \mathrm{E}$ \\
\hline
\end{tabular}




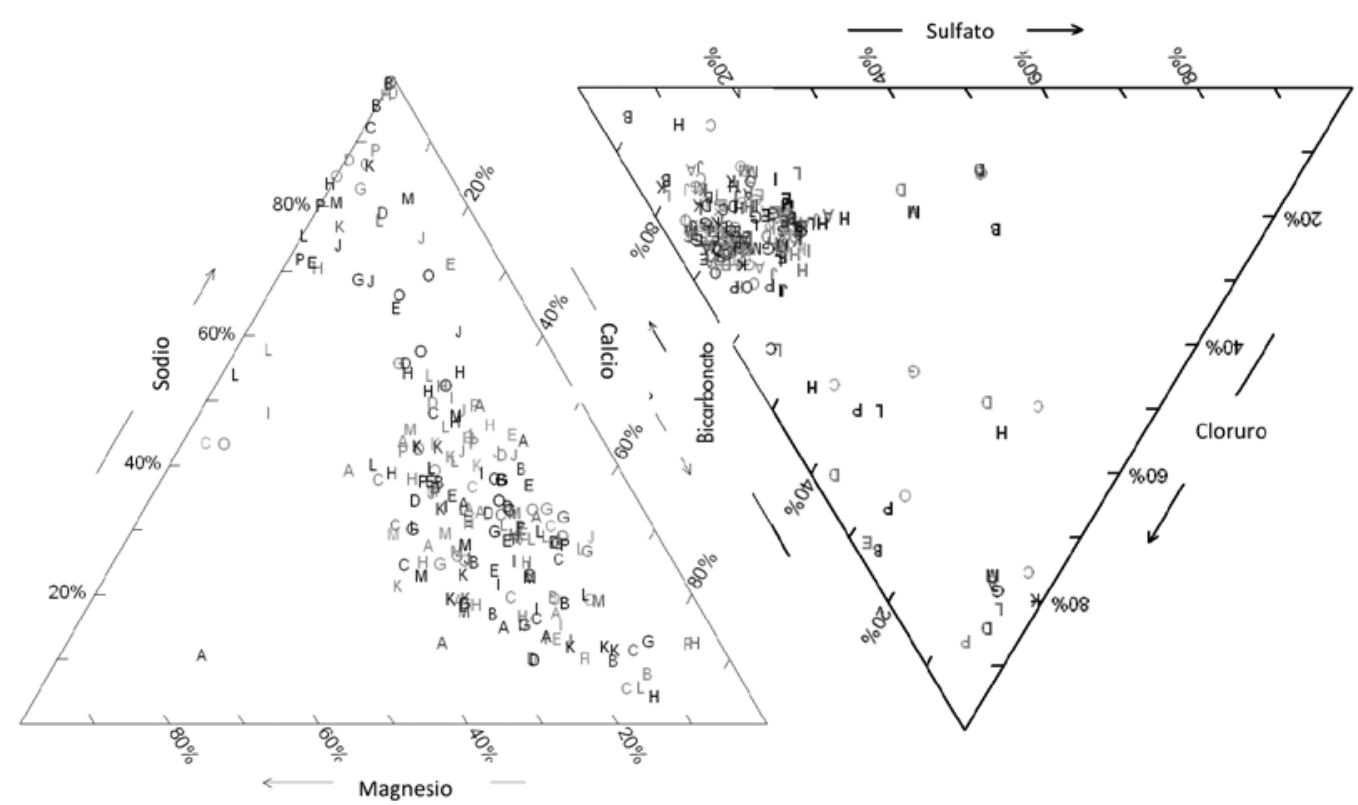

Figura 1. Composiciones químicas de las muestras de aguas superficiales de los estados de Tlaxcala, Puebla y Veracruz.

es necesario que se proceda a graficar la relación log $\mathrm{Na}\left(\mathrm{mmol} \mathrm{L}^{-1}\right)-\log \mathrm{Cl}\left(\mathrm{mmol} \mathrm{L}^{-1}\right)$. Esta forma de relacionar $\mathrm{Na}-\mathrm{Cl}$ nos permite identificar aguas que por su composición y concentración están en total correspondencia con los procesos permanentes de intemperismo con las rocas de las diferentes cuencas que estas aguas atraviesan, y además, la Figura 1 nos permite conocer aguas de alta concentración, en donde las sales de baja solubilidad ya se han precipitado; en este tipo de aguas predominarán las sales más solubles.

En las aguas que tienen altas concentraciones iónicas predominan los cloruros (Figura 2). El modelo de evolución de la composición química de las diferentes aguas, que se presenta a medida que aumenta la concentración conducirá a una precipitación de sales, que en su secuencia, estará en concordancia con los procesos de solubilidad. El primer compuesto que casi siempre se precipitará debido a su baja solubilidad será $\mathrm{CaCO}_{3}$. El esquema de la precipitación de la calcita se presenta de acuerdo con el producto de las actividades de los iones calcio $\left(\mathrm{Ca}^{2+}\right)$ y carbonato $\left(\mathrm{CO}_{3}{ }^{2-}\right)$, en la solución acuosa y este producto debe permanecer constante e igual al producto de la solubilidad de la calcita: $\left(\mathrm{Ca}^{2+}\right) \cdot\left(\mathrm{CO}_{3}{ }^{2-}\right)=\mathrm{K}$ calcita

Debido a que la calcita se precipita en intervalos de baja salinidad, los valores de las concentraciones pueden considerarse como valores de actividades, en una primera aproximación, entonces, debe quedar constante el valor del producto de las concentraciones del ion calcio $\mathrm{Ca}^{2+}$ y del ion carbonato $\mathrm{CO}_{3}{ }^{2-}$. Esto quiere decir que ambos iones no pueden concentrarse conjuntamente, de tal manera, si la concentración de uno de estos iones aumenta, la concentración del otro ion debe forzosamente bajar. Esta situación particular define las dos vías fundamentales de evolución de las distintas aguas que en proceso de migración aumentan su concentración. Si en la solución acuosa inicial se tienen más iones de calcio que iones carbonato, la solución acuosa al aumentar la concentración se enriquecerá con iones calcio y se empobrecerá de iones carbonato, manteniéndose el $\mathrm{pH}$ con valores menores de 9,0 , es la vía evolutiva geoquímica neutra. Por el contrario, si en la solución acuosa inicial se tiene una mayor concentración de iones de carbonato que iones de calcio, entonces se concentrará en la solución el ion carbonato y disminuirá como consecuencia la concentración de iones calcio, esta situación hace que se eleve el pH por encima del valor de 10,0. Esta es la vía evolutiva geoquímica alcalina que conduce a soluciones salinas del tipo $\mathrm{Na}-\mathrm{CO}_{3}-\left(\mathrm{SO}_{4}\right)-\mathrm{Cl}$.

Como se mencionó, la calcita es el primer mineral que comúnmente se precipita en las diferentes aguas que inician un determinado aumento de 


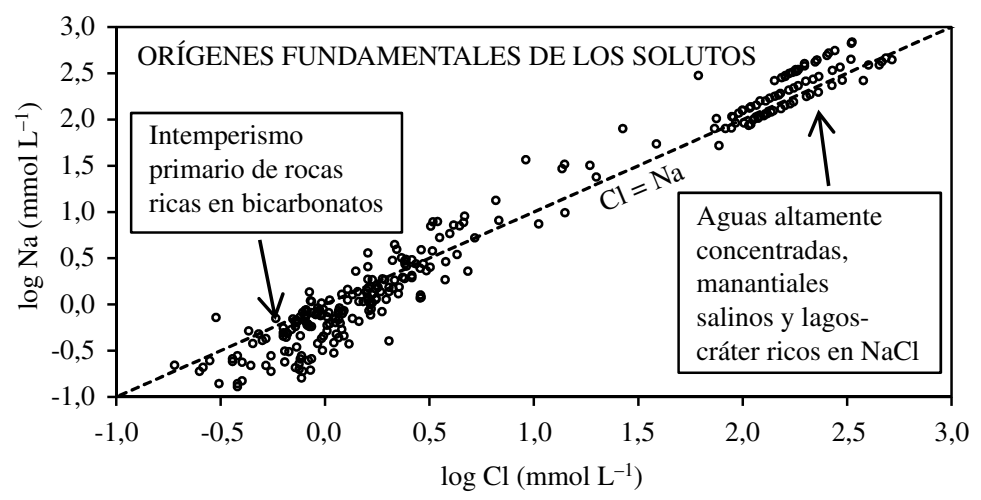

Figura 2. Concentraciones de sodio versus cloruro en las aguas que circulan en cuencas fluviales de escurrimiento y estancadas en los lagos-cráter.

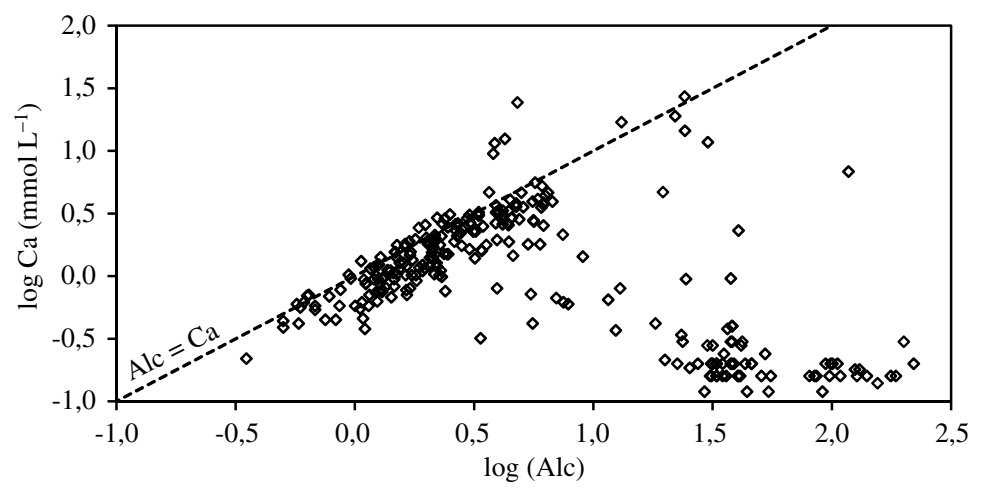

Figura 3. Concentración de calcio versus alcalinidad en las aguas superficiales de los estados de Tlaxcala, Puebla y Veracruz.

concentración. En la Figura 3 se presenta la relación $\log \mathrm{Ca}\left(\mathrm{mmol} \mathrm{L}^{-1}\right)$ y $\log (\mathrm{Alc})$ de las diferentes aguas de los tres muestreos; como se puede observar la gran mayoría de las aguas precipitarán a la calcita y tomarán por consiguiente la vía evolutiva de las aguas bicarbonatadas.

El efecto de la precipitación de las sales magnésicas sobre la evolución geoquímica de las diferentes aguas de los tres muestreos que durante los procesos de circulación aumentan de concentración, se puede observar en la Figura 4, en donde se grafica $\mathrm{Ca}+\mathrm{Mg}$ versus Alc. Las aguas cuya colocación se encuentra por debajo de la recta Alc $=\mathrm{Ca}+\mathrm{Mg}$ seguirán la vía evolutiva geoquímica carbonatada hasta llegar a ser soluciones en carbonato de sodio.

Por otra parte y continuando sobre el proceso sucesivo de aumento de las concentraciones en las aguas muestreadas en 2009, 2010 y 2011 se analiza el momento cuando en las soluciones se inicia la precipitación del yeso por la vía inicial "neutra" $\left(\mathrm{Ca}^{2+}\right.$ aumenta o [Ca] > [Alc]. En este punto las vías evolutivas son: a) vía evolutiva sulfatada neutra o bien, b) la vía evolutiva cálcica. En la Figura 5, se presenta la relación log $\mathrm{Ca}$ $\left(\mathrm{mmol}_{\mathrm{c}} \mathrm{L}^{-1}\right)-\log \mathrm{Alc}+\mathrm{SO}_{4}\left(\mathrm{mmol}_{\mathrm{c}} \mathrm{L}^{-1}\right)$. Aquí se comparan las concentraciones de $\mathrm{Ca}^{2+}$ y SO$_{4}{ }^{2-}$ al principio de la precipitación del yeso. Se observa que la recta $\mathrm{Ca}=\mathrm{Alc}+\mathrm{SO}_{4}$ separa exactamente a las aguas que siguen la vía evolutiva cálcica de aquellas aguas que siguen la vía evolutiva sulfatada. Esto significa que la relación Alc + $\mathrm{SO}_{4} / \mathrm{Ca}$ del agua inicial nos permite determinar si una agua cualquiera seguirá la vía evolutiva cálcica o la vía evolutiva sulfatada neutra cuando precipite el yeso de las soluciones.

Los resultados analíticos y determinaciones de las sales hipotéticas de los procesos de evaporación de las aguas salinas del lago-cráter Alchichica se presentan en la Tabla 3 y Figura 6. Para una representación gráfica del comportamiento sucesivo 


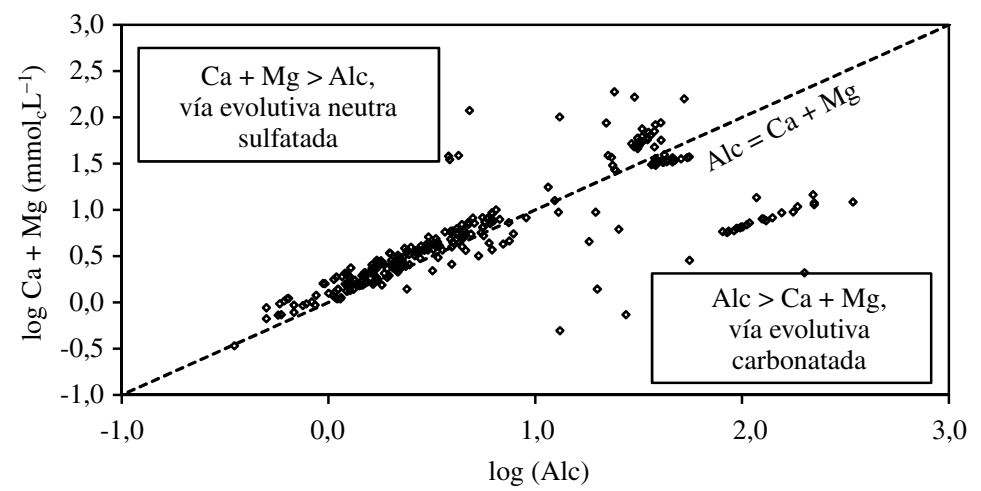

Figura 4. Relación de concentraciones de $\mathrm{Ca}+\mathrm{Mg}$ versus alcalinidad $\left(\mathrm{mmol}_{\mathrm{c}} \mathrm{L}^{-1}\right)$, en las aguas de los tres muestreos.

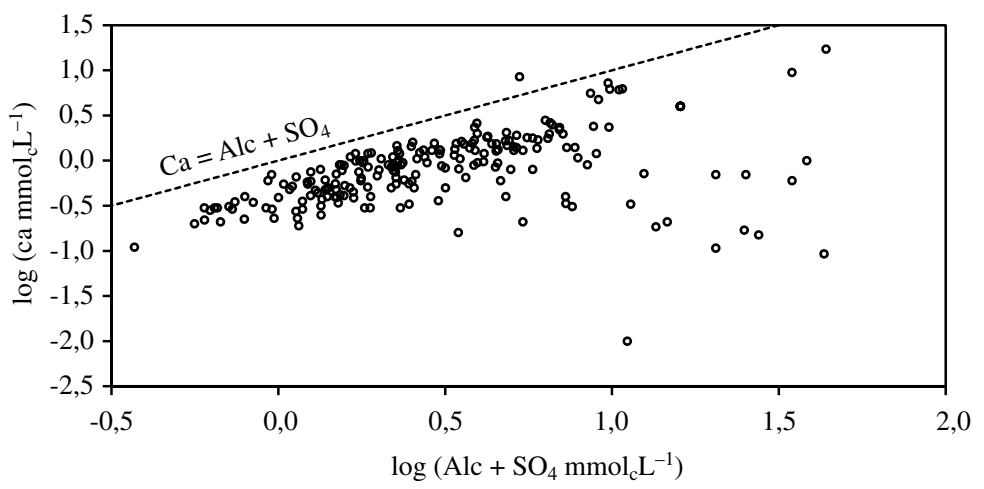

Figura 5. Concentraciones de Ca versus Alcalinidad $+\mathrm{SO}_{4},\left(\mathrm{mmol}_{\mathrm{c}} \mathrm{L}^{-1}\right)$, en las aguas de los tres muestreos.

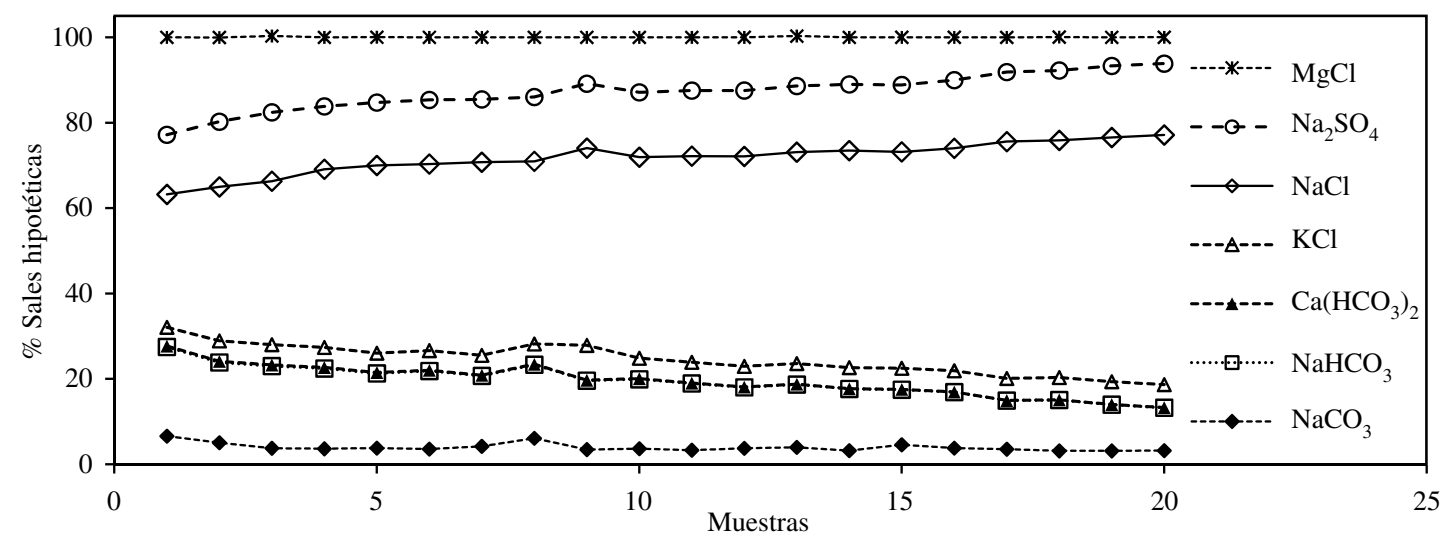

Figura 6. Sales hipotéticas expresadas en porcentaje, de las aguas evaporadas del lago-cráter Alchichica.

de la evaporación se eligió el diagrama de Durov (Durov, 1948; Zaporozec, 1972), (Figura 7). Los resultados analíticos y determinación de las sales hipotéticas de las aguas salinas del lago cráter Atexcac, se presentan en la Tabla 4 y Figura 8. Para una representación gráfica del comportamiento sucesivo de la evaporación se eligió el diagrama de Durov (Figura 9). Los resultados analíticos y determinación de las sales hipotéticas de las aguas salinas del manantial en Zapotitlán Salinas, 
Tabla 3. Composición iónica de los procesos de metamorfosis y evolución de las aguas del lago-cráter Alchichica, Puebla.

\begin{tabular}{|c|c|c|c|c|c|c|c|c|c|c|c|c|c|c|c|}
\hline \multirow{2}{*}{$\mathbf{N}^{o}$} & \multirow{2}{*}{ pH } & \multirow{2}{*}{$\begin{array}{c}\mathrm{CE} \\
\mu \mathrm{S} \mathrm{\text {cm}^{-1 }}\end{array}$} & $\mathrm{Ca}^{2+}$ & $\mathbf{M g}^{2+}$ & $\mathrm{Na}^{+}$ & $\mathbf{K}^{+}$ & \multirow{2}{*}{ Suma } & $\mathrm{CO}_{3}{ }^{2-}$ & $\mathrm{HCO}_{3}^{-}$ & $\mathrm{Cl}^{-}$ & $\mathrm{SO}_{4}{ }^{2-}$ & \multirow{2}{*}{ Suma } & \multirow{2}{*}{$\begin{array}{c}\% \\
\text { error }\end{array}$} & \multirow{2}{*}{$\begin{array}{c}\mathrm{mg} \mathrm{L}^{-1} \\
\mathrm{mmol} \mathrm{L}^{-1}\end{array}$} & \multirow{2}{*}{$\begin{array}{c}\text { RSE } \\
\mathrm{mg} \mathrm{L}^{-1}\end{array}$} \\
\hline & & & \multicolumn{4}{|c|}{ mmolc $L^{-1}$} & & \multicolumn{4}{|c|}{ mmolc $\mathrm{L}^{-1}$} & & & & \\
\hline 1 & 8,5 & 15850 & 0,30 & 39,50 & 107,80 & 6,70 & 154,30 & 10,00 & 32,00 & 88,50 & 21,20 & 151,70 & 1,45 & & 9828,22 \\
\hline & & & 6,01 & 479,80 & 2479,40 & 261,60 & & 300,00 & 1952,30 & 3138,60 & 1017,80 & & & 9635,50 & \\
\hline & & & 0,15 & 19,70 & 107,80 & 6,70 & & 5,00 & 32,00 & 88,50 & 10,60 & & & 270,50 & \\
\hline 2 & 8,7 & 16410 & 0,40 & 35,70 & 117,40 & 7,60 & 161,10 & 8,00 & 30,00 & 95,40 & 24,10 & 157,50 & 1,96 & & 10146,10 \\
\hline & & & 8,02 & 434,00 & 2700,70 & 295,20 & & 240,00 & 1830,30 & 3381,80 & 1155,60 & & & 10045,60 & \\
\hline & & & 0,20 & 17,80 & 117,40 & 7,60 & & 4,00 & 30,00 & 95,40 & 12,00 & & & 284,40 & \\
\hline 3 & 8,8 & 17010 & 0,40 & 34,10 & 125,20 & 7,30 & 167,00 & 6,20 & 32,00 & 99,50 & 26,50 & 164,20 & 1,43 & & 10843,70 \\
\hline & & & 8,02 & 414,80 & 2878,50 & 286,60 & & 186,00 & 1952,30 & 3529,30 & 1272,30 & & & 10527,80 & \\
\hline & & & 0,20 & 17,10 & 125,20 & 7,30 & & 3,10 & 32,00 & 99,50 & 13,20 & & & 297,60 & \\
\hline 4 & 8,9 & 17510 & 0,40 & 32,00 & 126,60 & 7,90 & 166,90 & 6,10 & 32,00 & 99,50 & 26,50 & 164,10 & 1,44 & & 10766,50 \\
\hline & & & 8,02 & 389,40 & 2912,00 & 308,50 & & 183,60 & 1952,30 & 3529,30 & 1272,30 & & & 10555,40 & \\
\hline & & & 0,20 & 16,00 & 126,60 & 7,90 & & 3,10 & 32,00 & 99,50 & 13,20 & & & 298,50 & \\
\hline 5 & 9,0 & 17590 & 0,38 & 30,60 & 133,10 & 7,60 & 171,68 & 6,40 & 30,00 & 107,50 & 24,90 & 168,80 & 1,49 & & 10876,40 \\
\hline & & & 7,62 & 372,00 & 3061,50 & 298,30 & & 192,60 & 1830,30 & 3810,50 & 1195,90 & & & 10768,70 & \\
\hline & & & 0,19 & 15,30 & 133,10 & 7,60 & & 3,20 & 30,00 & 107,50 & 12,50 & & & 309,40 & \\
\hline 6 & 8,8 & 18040 & 0,30 & 30,20 & 137,60 & 8,00 & 176,10 & 6,20 & 32,00 & 109,20 & 26,00 & 173,40 & 1,39 & & 11442,40 \\
\hline & & & 6,01 & 367,70 & 3165,50 & 312,40 & & 185,40 & 1952,30 & 3870,50 & 1249,30 & & & 11109,10 & \\
\hline & & & 0,15 & 15,10 & 137,60 & 8,00 & & 3,10 & 32,00 & 109,20 & 13,00 & & & 318,20 & \\
\hline 7 & 8,9 & 18700 & 0,30 & 30,80 & 143,20 & 8,40 & 182,70 & 7,60 & 30,00 & 115,90 & 26,50 & 180,00 & 1,26 & & 11672,40 \\
\hline & & & 6,01 & 374,60 & 3294,10 & 326,50 & & 228,00 & 1830,30 & 4111,20 & 1272,80 & & & 11443,50 & \\
\hline & & & 0,15 & 15,40 & 143,20 & 8,40 & & 3,80 & 30,00 & 115,90 & 13,30 & & & 330,20 & \\
\hline 8 & 8,9 & 20420 & 0,20 & 33,00 & 157,40 & 9,40 & 200,00 & 12,00 & 34,00 & 120,80 & 29,60 & 196,40 & 1,53 & & 12698,20 \\
\hline & & & 4,01 & 400,80 & 3665,10 & 365,60 & & 360,00 & 2074,30 & 4282,50 & 1420,20 & & & 12572,50 & \\
\hline & & & 0,10 & 16,50 & 159,40 & 9,40 & & 6,00 & 34,00 & 120,80 & 14,80 & & & 361,00 & \\
\hline 9 & 8,6 & 20500 & 0,20 & 33,50 & 157,30 & 9,50 & 200,50 & 6,80 & 32,00 & 128,50 & 29,70 & 197,00 & 1,47 & & 12917,80 \\
\hline & & & 4,01 & 407,20 & 3617,20 & 371,10 & & 204,00 & 1952,30 & 4557,30 & 1428,40 & & & 12541,50 & \\
\hline & & & 0,10 & 16,70 & 157,30 & 9,50 & & 3,40 & 32,00 & 128,50 & 14,90 & & & 362,40 & \\
\hline 10 & 8,8 & 21580 & 0,28 & 32,40 & 168,40 & 10,00 & 211,08 & 7,60 & 34,00 & 134,30 & 31,50 & 207,40 & 1,53 & & 13505,90 \\
\hline & & & 5,61 & 394,50 & 3874,10 & 389,40 & & 228,00 & 2074,30 & 4762,30 & 1512,90 & & & 13241,10 & \\
\hline & & & 0,14 & 16,20 & 168,40 & 10,00 & & 3,80 & 34,00 & 134,30 & 15,80 & & & 382,60 & \\
\hline 11 & 8,9 & 22530 & 0,16 & 32,90 & 176,90 & 10,60 & 220,56 & 7,20 & 34,00 & 142,20 & 33,30 & 216,70 & 1,52 & & 13999,50 \\
\hline & & & 3,21 & 399,60 & 4114,50 & 414,10 & & 216,00 & 2074,30 & 5041,70 & 1597,50 & & & 13860,90 & \\
\hline & & & 0,08 & 16,40 & 178,90 & 10,60 & & 3,60 & 34,00 & 142,20 & 16,60 & & & 402,40 & \\
\hline 12 & 8,9 & 23210 & 0,16 & 33,80 & 182,40 & 10,80 & 227,16 & 8,40 & 32,00 & 148,30 & 34,50 & 223,20 & 1,54 & & 14576,50 \\
\hline & & & 3,21 & 411,30 & 4195,70 & 422,70 & & 252,00 & 1952,30 & 5260,10 & 1654,60 & & & 14151,90 & \\
\hline & & & 0,08 & 16,90 & 182,40 & 10,80 & & 4,20 & 32,00 & 148,30 & 17,20 & & & 411,90 & \\
\hline 13 & 8,9 & 23920 & 0,20 & 33,10 & 189,80 & 11,10 & 234,20 & 9,20 & 34,00 & 151,20 & 35,60 & 230,00 & 1,56 & & 14921,10 \\
\hline & & & 4,01 & 402,70 & 4365,40 & 434,40 & & 276,00 & 2074,30 & 5362,30 & 1709,40 & & & 14628,50 & \\
\hline & & & 0,10 & 16,60 & 189,80 & 11,10 & & 4,60 & 34,00 & 151,20 & 17,80 & & & 425,20 & \\
\hline 14 & 8,9 & 25870 & 0,12 & 34,40 & 206,70 & 12,30 & 253,52 & 8,00 & 36,00 & 165,90 & 38,60 & 248,50 & 1,72 & & 15984,00 \\
\hline & & & 2,40 & 418,40 & 4753,20 & 480,50 & & 240,00 & 2196,40 & 5883,20 & 1851,60 & & & 15825,70 & \\
\hline & & & 0,06 & 17,20 & 206,70 & 12,30 & & 4,00 & 36,00 & 165,90 & 19,30 & & & 461,50 & \\
\hline 15 & 9,0 & 27280 & 0,20 & 36,60 & 217,70 & 13,00 & 267,50 & 12,00 & 34,00 & 175,00 & 41,10 & 262,10 & 1,72 & & 17072,70 \\
\hline & & & 4,01 & 444,70 & 5006,20 & 507,50 & & 360,00 & 2074,30 & 6206,60 & 1972,10 & & & 16575,40 & \\
\hline & & & 0,10 & 18,30 & 217,70 & 13,00 & & 6,00 & 34,00 & 175,00 & 20,50 & & & 484,60 & \\
\hline 16 & 8,9 & 28550 & 0,04 & 35,40 & 229,80 & 13,60 & 278,84 & 10,40 & 36,00 & 183,90 & 43,70 & 274,00 & 1,54 & & 17727,30 \\
\hline & & & 0,80 & 430,80 & 5286,30 & 533,30 & & 312,00 & 2196,40 & 6519,70 & 2100,40 & & & 17379,70 & \\
\hline & & & 0,02 & 17,70 & 229,80 & 13,60 & & 5,20 & 36,00 & 183,90 & 21,90 & & & 508,10 & \\
\hline 17 & 8,8 & 32810 & 0,08 & 34,40 & 270,30 & 16,30 & 321,08 & 11,20 & 36,00 & 216,70 & 51,30 & 315,20 & 1,55 & & 20154,30 \\
\hline & & & 1,60 & 418,40 & 6215,80 & 636,50 & & 336,00 & 2196,40 & 7684,20 & 2465,90 & & & 19954,80 & \\
\hline & & & 0,04 & 17,20 & 270,30 & 16,30 & & 5,60 & 36,00 & 216,70 & 25,70 & & & 587,80 & \\
\hline 18 & 8,8 & 35010 & 0,16 & 35,60 & 289,40 & 17,60 & 342,76 & 10,60 & 40,00 & 230,80 & 55,10 & 336,50 & 1,57 & & 22009,80 \\
\hline & & & 3,21 & 432,70 & 6656,90 & 687,40 & & 318,00 & 2440,40 & 8185,60 & 2644,50 & & & 21368,70 & \\
\hline & & & 0,08 & 17,80 & 289,40 & 17,60 & & 5,30 & 40,00 & 230,80 & 27,50 & & & 628,50 & \\
\hline 19 & 8,8 & 40300 & 0,12 & 36,70 & 337,40 & 20,70 & 394,92 & 12,20 & 42,00 & 268,10 & 64,90 & 387,20 & 1,70 & & 25063,80 \\
\hline & & & 2,40 & 446,80 & 7760,70 & 810,20 & & 366,00 & 2562,40 & 9506,80 & 3117,10 & & & 24572,40 & \\
\hline & & & 0,06 & 18,40 & 337,40 & 20,70 & & 6,10 & 42,00 & 268,10 & 32,50 & & & 725,30 & \\
\hline 20 & 8,8 & 43400 & 0,16 & 37,40 & 365,60 & 22,40 & 425,56 & 13,40 & 42,00 & 291,90 & 69,70 & 417,00 & 1,74 & & 26670,70 \\
\hline & & & 3,21 & 454,50 & 8409,50 & 877,00 & & 402,00 & 2562,40 & 10352,20 & 3345,80 & & & 26406,60 & \\
\hline & & & 0,08 & 18,70 & 365,60 & 22,40 & & 6,70 & 42,00 & 291,90 & 34,80 & & & 782,20 & \\
\hline
\end{tabular}




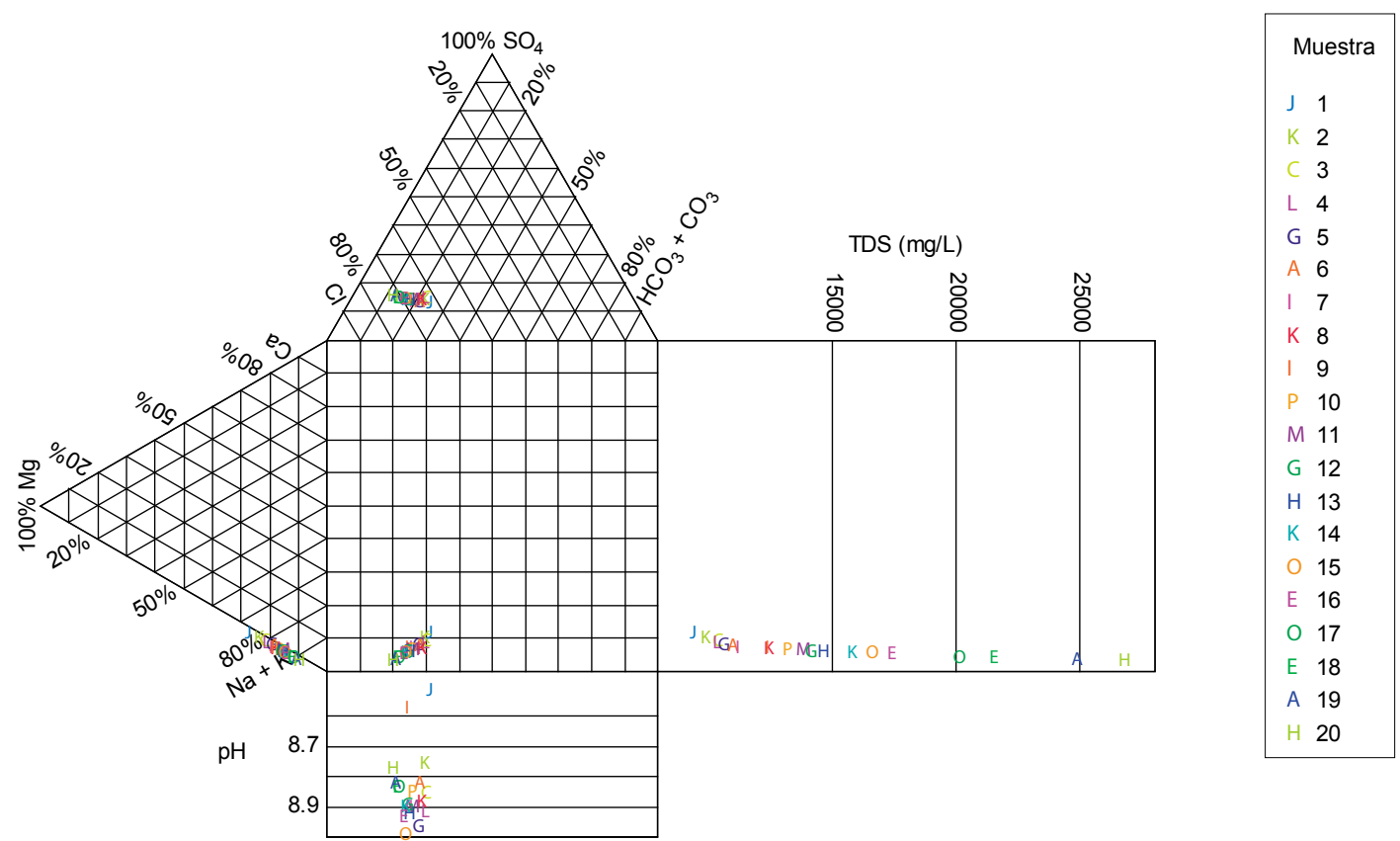

Figura 7. Diagrama de Durov, para las aguas del lago-cráter Alchichica.

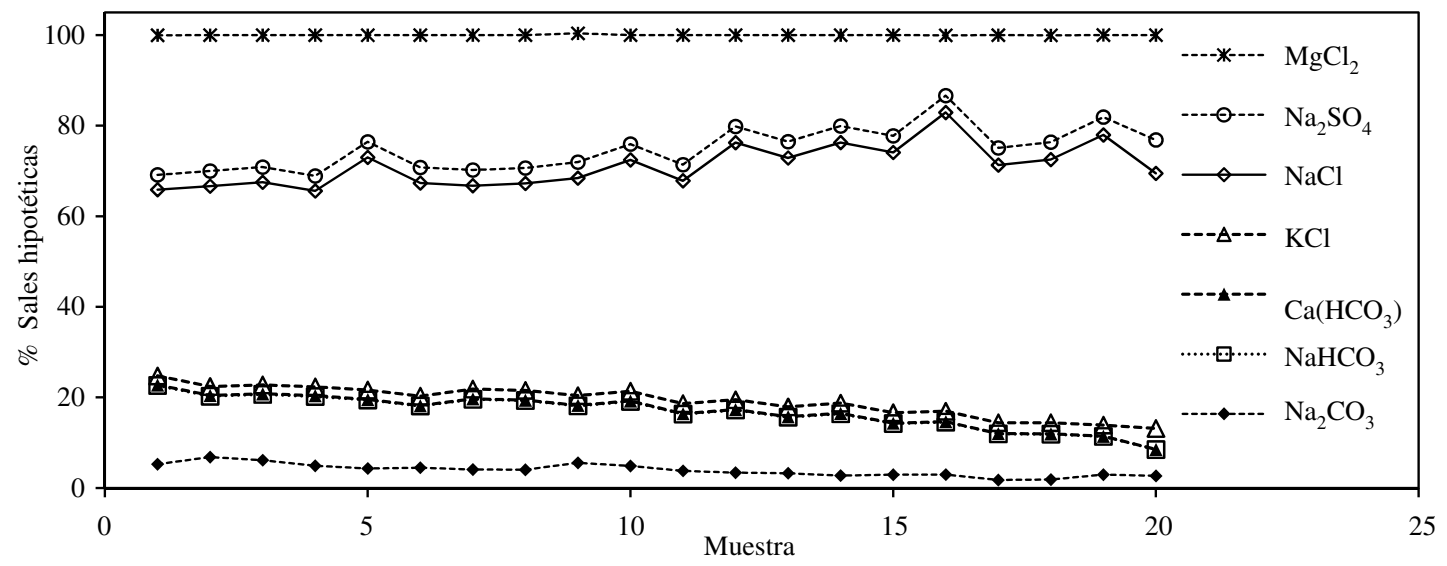

Figura 8. Sales hipotéticas, en porcentaje, de las aguas evaporadas del lago-cráter Atexcac.

se presentan en la Tabla 5 y Figura 10. Para una representación gráfica del comportamiento sucesivo de la evaporación se eligió el diagrama de Durov (Figura 11).

En las aguas de Alchichica y Atexcac en el proceso de sucesión de las sales hipotéticas, se observa que el cloruro de magnesio $\left(\mathrm{MgCl}_{2}\right)$ disminuye. Este comportamiento de disminución del cloruro de magnesio a medida que aumenta la concentración, se debe a la precipitación parcial del magnesio con los compuestos del silicio y del ion carbonato. La precipitación de las sales del ion magnesio se precipitan como sigue: Después de la precipitación de la calcita se precipitan los silicatos o carbonatos de magnesio; que son sales poco solubles. En la vía geoquímica alcalina, el aumento del $\mathrm{pH}$ induce la precipitación de silicatos de magnesio (Badaut y Risacher, 1983), según la reacción general siguiente: $\mathrm{Mg}^{2+}+\mathrm{nH}_{4} \mathrm{SiO}_{4} \rightarrow$ $\mathrm{Mg}$-Silicato $+2 \mathrm{H}^{+}$y $\mathrm{Mg}^{2+} \mathrm{CO}_{3}^{2-} \rightarrow \mathrm{MgCO}_{3}$. 
Tabla 4. Composición iónica de los procesos de metamorfosis y evolución de las aguas del lago-cráter Atexcac, de la cuenca del Oriental, Puebla.

\begin{tabular}{|c|c|c|c|c|c|c|c|c|c|c|c|c|c|c|c|}
\hline \multirow{2}{*}{$\mathbf{N}^{\circ}$} & \multirow{2}{*}{ pH } & \multirow{2}{*}{$\begin{array}{c}\text { CE } \\
\mu \mathrm{S} \mathrm{cm}^{-1} \\
\end{array}$} & $\mathrm{Ca}^{2+}$ & $\mathrm{Mg}^{2+}$ & $\mathrm{Na}^{+}$ & $\mathbf{K}^{+}$ & \multirow{2}{*}{ Suma } & $\mathrm{CO}_{3}{ }^{2-}$ & $\mathrm{HCO}_{3}^{-}$ & $\mathrm{Cl}^{-}$ & $\mathrm{SO}_{4}^{2-}$ & \multirow{2}{*}{ Suma } & \multirow{2}{*}{$\begin{array}{c}\% \\
\text { error }\end{array}$} & \multirow{2}{*}{$\begin{array}{c}\mathrm{mg} \mathrm{L}^{-1} \\
\mathbf{m m o l ~ L}^{-1}\end{array}$} & \multirow{2}{*}{$\begin{array}{c}\text { RSE } \\
\text { mg L }^{-1}\end{array}$} \\
\hline & & & \multicolumn{4}{|c|}{ mmolc $\mathrm{L}^{-1}$} & & & mmo & Ic $\mathrm{L}^{-1}$ & & & & & \\
\hline \multirow[t]{3}{*}{1} & 8,8 & 14260 & 0,20 & 46,10 & 89,90 & 2,70 & 138,90 & 7,20 & 24,00 & 101,30 & 4,50 & 137,00 & 1,32 & & 8470,00 \\
\hline & & & 3,20 & 560,70 & 2066,80 & 106,00 & & 216,00 & 1464,20 & 3590,30 & 216,10 & & & 8223,30 & \\
\hline & & & 0,10 & 23,10 & 89,90 & 2,70 & & 3,60 & 24,00 & 101,30 & 2,30 & & & 247,00 & \\
\hline 2 & 9,0 & 15270 & 0,30 & 48,10 & 97,50 & 2,90 & 148,80 & 10,00 & 20,00 & 111,80 & 4,90 & 146,70 & 1,38 & & 8751,95 \\
\hline & & & 5,60 & 585,40 & 2242,50 & 112,60 & & 300,00 & 1220,20 & 3963,70 & 235,30 & & & 8665,30 & \\
\hline & & & 0,10 & 24,10 & 97,50 & 2,90 & & 5,00 & 20,00 & 111,80 & 2,50 & & & 263,90 & \\
\hline 3 & 9,0 & 15680 & 0,20 & 48,60 & 101,20 & 2,90 & 152,90 & 9,20 & 22,00 & 113,80 & 5,00 & 150,00 & 1,80 & & 9109,01 \\
\hline & & & 4,00 & 590,50 & 2326,50 & 115,00 & & 276,00 & 1342,20 & 4034,60 & 241,60 & & & 8930,40 & \\
\hline & & & 0,10 & 24,30 & 101,20 & 2,90 & & 4,60 & 22,00 & 113,80 & 2,50 & & & 271,40 & \\
\hline 4 & 8,9 & 15770 & 0,30 & 49,00 & 101,50 & 3,00 & 153,80 & 7,60 & 24,00 & 118,30 & 5,10 & 155,00 & 0,79 & & 9461,07 \\
\hline & & & 5,60 & 595,20 & 2334,30 & 118,10 & & 228,00 & 1464,20 & 4194,20 & 245,90 & & & 9185,50 & \\
\hline & & & 0,10 & 24,50 & 101,50 & 3,00 & & 3,80 & 24,00 & 118,30 & 2,60 & & & 277,80 & \\
\hline 5 & 8,8 & 16450 & 0,20 & 52,00 & 101,00 & 3,30 & 156,50 & 6,80 & 24,00 & 118,30 & 5,10 & 154,20 & 1,39 & & 9288,06 \\
\hline & & & 3,20 & 632,00 & 2323,20 & 129,40 & & 204,00 & 1464,20 & 4194,20 & 245,90 & & & 9196,10 & \\
\hline & & & 0,10 & 26,00 & 101,00 & 3,30 & & 3,40 & 24,00 & 118,30 & 2,60 & & & 278,70 & \\
\hline 6 & 8,8 & 16760 & 0,10 & 52,00 & 108,20 & 3,40 & 163,70 & 7,20 & 22,00 & 125,90 & 5,50 & 160,60 & 1,82 & & 9729,88 \\
\hline & & & 2,40 & 632,40 & 2487,50 & 131,40 & & 216,00 & 1342,20 & 4463,00 & 264,20 & & & 9539,10 & \\
\hline & & & 0,10 & 26,00 & 108,20 & 3,40 & & 3,60 & 22,00 & 125,90 & 2,80 & & & 292,00 & \\
\hline 7 & 8,8 & 17380 & 0,20 & 54,60 & 110,60 & 3,60 & 169,00 & 6,80 & 26,00 & 128,10 & 5,80 & 166,70 & 1,33 & & 10262,10 \\
\hline & & & 3,20 & 664,10 & 2544,30 & 141,50 & & 204,00 & 1586,30 & 4543,10 & 276,70 & & & 9963,20 & \\
\hline & & & 0,10 & 27,30 & 110,60 & 3,60 & & 3,40 & 26,00 & 128,10 & 2,90 & & & 302,00 & \\
\hline 8 & 8,7 & 17620 & 0,20 & 54,60 & 113,60 & 3,60 & 172,00 & 6,80 & 26,00 & 130,40 & 5,80 & 169,00 & 1,70 & & 10215,20 \\
\hline & & & 3,20 & 664,10 & 2613,30 & 141,50 & & 204,00 & 1586,30 & 4625,00 & 276,70 & & & 10114,10 & \\
\hline & & & 0,10 & 27,30 & 113,60 & 3,60 & & 3,40 & 26,00 & 130,40 & 2,90 & & & 307,30 & \\
\hline 9 & 8,8 & 18130 & 0,20 & 54,40 & 118,70 & 3,70 & 177,00 & 9,60 & 22,00 & 136,30 & 6,10 & 174,00 & 1,69 & & 10502,40 \\
\hline & & & 4,00 & 661,40 & 2731,00 & 145,50 & & 288,00 & 1342,20 & 4831,40 & 293,00 & & & 10296,50 & \\
\hline & & & 0,10 & 27,20 & 118,70 & 3,70 & & 4,80 & 22,00 & 136,30 & 3,10 & & & 315,90 & \\
\hline 10 & 8,8 & 18840 & 0,20 & 57,40 & 122,60 & 3,90 & 184,10 & 8,80 & 26,00 & 139,80 & 6,30 & 180,90 & 1,63 & & 11108,80 \\
\hline & & & 4,00 & 698,30 & 2818,70 & 151,70 & & 264,00 & 1586,30 & 4958,70 & 303,50 & & & 10785,20 & \\
\hline & & & 0,10 & 28,70 & 122,60 & 3,90 & & 4,40 & 26,00 & 139,80 & 3,20 & & & 328,70 & \\
\hline 11 & 8,7 & 19790 & 0,20 & 59,80 & 129,30 & 4,20 & 193,50 & 7,20 & 24,00 & 152,10 & 6,80 & 190,10 & 1,70 & & 11378,40 \\
\hline & & & 4,00 & 726,70 & 2973,70 & 163,80 & & 216,00 & 1464,20 & 5393,10 & 324,20 & & & 11265,70 & \\
\hline & & & 0,10 & 29,90 & 129,30 & 4,20 & & 3,60 & 24,00 & 152,10 & 3,40 & & & 346,60 & \\
\hline 12 & 8,7 & 20930 & 0,20 & 60,30 & 139,90 & 4,40 & 204,80 & 6,80 & 28,00 & 159,00 & 7,20 & 201,00 & 1,77 & & 12259,00 \\
\hline & & & 3,20 & 732,90 & 3217,90 & 170,50 & & 204,00 & 1708,30 & 5637,40 & 344,40 & & & 12018,60 & \\
\hline & & & 0,10 & 30,10 & 139,90 & 4,40 & & 3,40 & 28,00 & 159,00 & 3,60 & & & 368,50 & \\
\hline 13 & 8,6 & 21740 & 0,20 & 62,00 & 144,90 & 4,60 & 211,70 & 6,80 & 26,00 & 168,30 & 7,50 & 208,60 & 1,44 & & 12758,00 \\
\hline & & & 4,00 & 754,20 & 3332,90 & 178,70 & & 204,00 & 1586,30 & 5966,10 & 360,20 & & & 12386,40 & \\
\hline & & & 0,10 & 31,00 & 144,90 & 4,60 & & 3,40 & 26,00 & 168,30 & 3,80 & & & 382,10 & \\
\hline 14 & 8,6 & 22760 & 0,20 & 64,00 & 152,60 & 5,00 & 221,80 & 6,00 & 30,00 & 174,70 & 8,00 & 218,70 & 1,37 & & 13203,10 \\
\hline & & & 3,20 & 778,20 & 3510,70 & 194,30 & & 180,00 & 1830,30 & 6193,40 & 382,30 & & & 13072,40 & \\
\hline & & & 0,10 & 32,00 & 152,60 & 5,00 & & 3,00 & 30,00 & 174,70 & 4,00 & & & 401,40 & \\
\hline 15 & 8,6 & 25530 & 0,20 & 68,80 & 174,90 & 5,60 & 249,50 & 7,20 & 28,00 & 201,40 & 9,00 & 245,60 & 1,55 & & 14875,50 \\
\hline & & & 4,80 & 837,00 & 4022,70 & 220,50 & & 216,00 & 1708,30 & 7141,30 & 433,20 & & & 14583,80 & \\
\hline & & & 0,10 & 34,40 & 174,90 & 5,60 & & 3,60 & 28,00 & 201,40 & 4,50 & & & 452,50 & \\
\hline 16 & 8,6 & 26700 & 0,20 & 70,90 & 184,60 & 6,00 & 261,70 & 7,60 & 30,00 & 209,70 & 9,60 & 256,90 & 1,77 & & 15756,30 \\
\hline & & & 4,00 & 862,30 & 4245,30 & 232,60 & & 228,00 & 1830,30 & 7435,30 & 459,60 & & & 15297,40 & \\
\hline & & & 0,10 & 35,50 & 184,60 & 6,00 & & 3,80 & 30,00 & 209,70 & 4,80 & & & 474,50 & \\
\hline 17 & 8,5 & 28360 & 0,20 & 75,20 & 195,10 & 6,50 & 277,00 & 4,80 & 28,00 & 229,70 & 10,30 & 272,80 & 1,48 & & 16316,20 \\
\hline & & & 4,00 & 914,60 & 4488,20 & 254,90 & & 144,00 & 1708,30 & 8145,50 & 495,20 & & & 16154,70 & \\
\hline & & & 0,10 & 37,60 & 195,10 & 6,50 & & 2,40 & 28,00 & 229,70 & 5,20 & & & 504,60 & \\
\hline 18 & 8,6 & 32960 & 0,20 & 83,20 & 231,30 & 7,80 & 322,50 & 6,00 & 32,00 & 266,80 & 12,20 & 317,00 & 1,62 & & 19199,50 \\
\hline & & & 4,00 & 1012,00 & 5318,80 & 306,20 & & 180,00 & 1952,30 & 9461,80 & 587,90 & & & 18823,00 & \\
\hline & & & 0,10 & 41,60 & 231,30 & 7,80 & & 3,00 & 32,00 & 266,80 & 6,10 & & & 588,70 & \\
\hline 19 & 8,5 & 36780 & 0,20 & 87,80 & 262,60 & 8,70 & 359,30 & 10,40 & 30,00 & 299,00 & 14,00 & 353,40 & 1,56 & & 21493,30 \\
\hline & & & 3,20 & 1067,80 & 6039,10 & 339,80 & & 312,00 & 1830,30 & 10603,60 & 671,50 & & & 20867,30 & \\
\hline & & & 0,10 & 43,90 & 262,60 & 8,70 & & 5,20 & 30,00 & 299,00 & 7,00 & & & 656,50 & \\
\hline 20 & 8,1 & 64000 & 0,20 & 159,20 & 439,10 & 28,40 & 626,90 & 16,40 & 36,00 & 517,30 & 45,50 & 615,20 & 1,76 & & 36729,90 \\
\hline & & & 4,80 & 1936,10 & 10100,00 & 1110,80 & & 492,00 & 2196,40 & 18342,70 & 2183,40 & & & 36366,20 & \\
\hline & & & 0,10 & 79,60 & 439,10 & 28,40 & & 8,20 & 36,00 & 517,30 & 22,70 & & & 1131,40 & \\
\hline
\end{tabular}




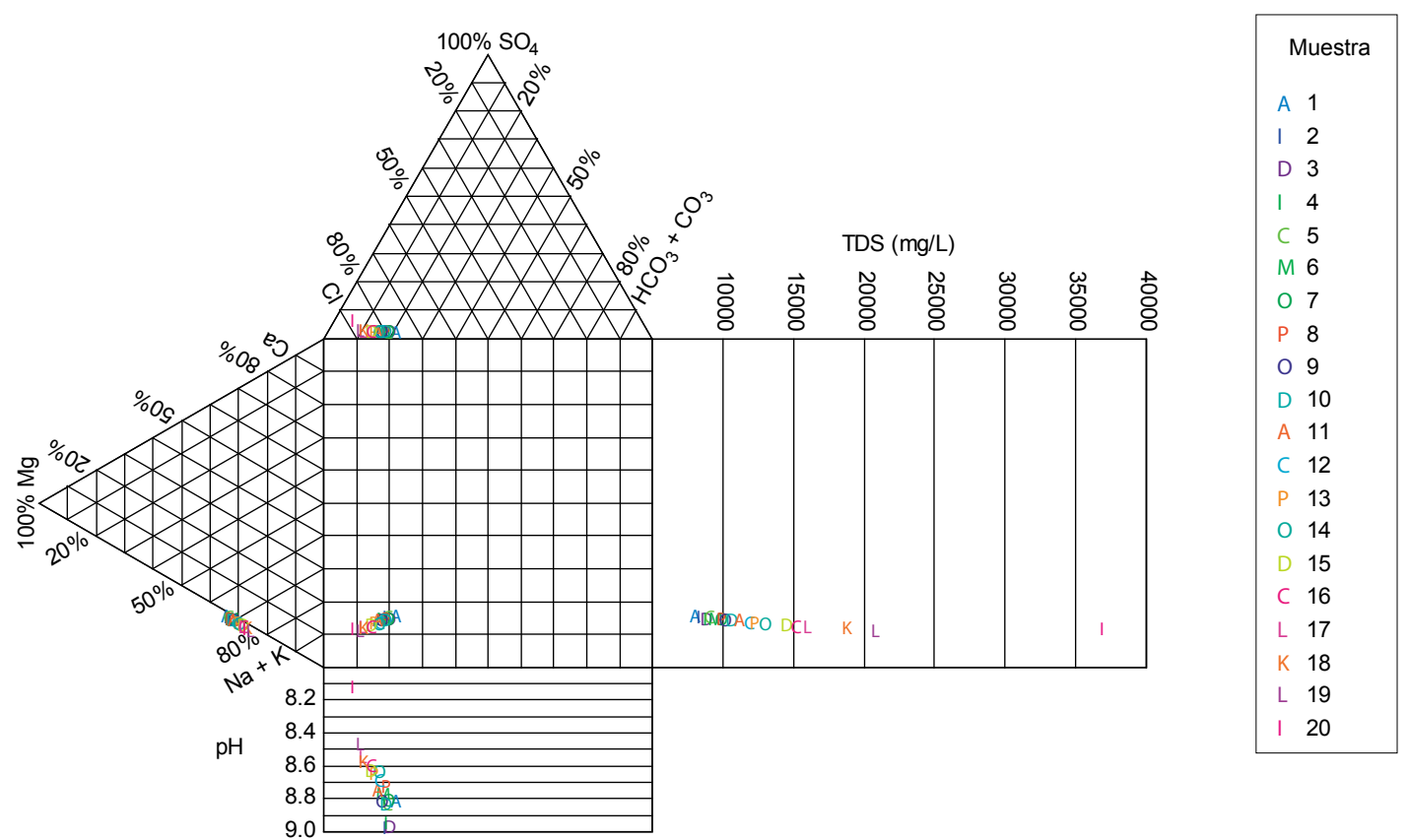

Figura 9. Diagrama de Durov, para las aguas del lago-cráter Atexcac.

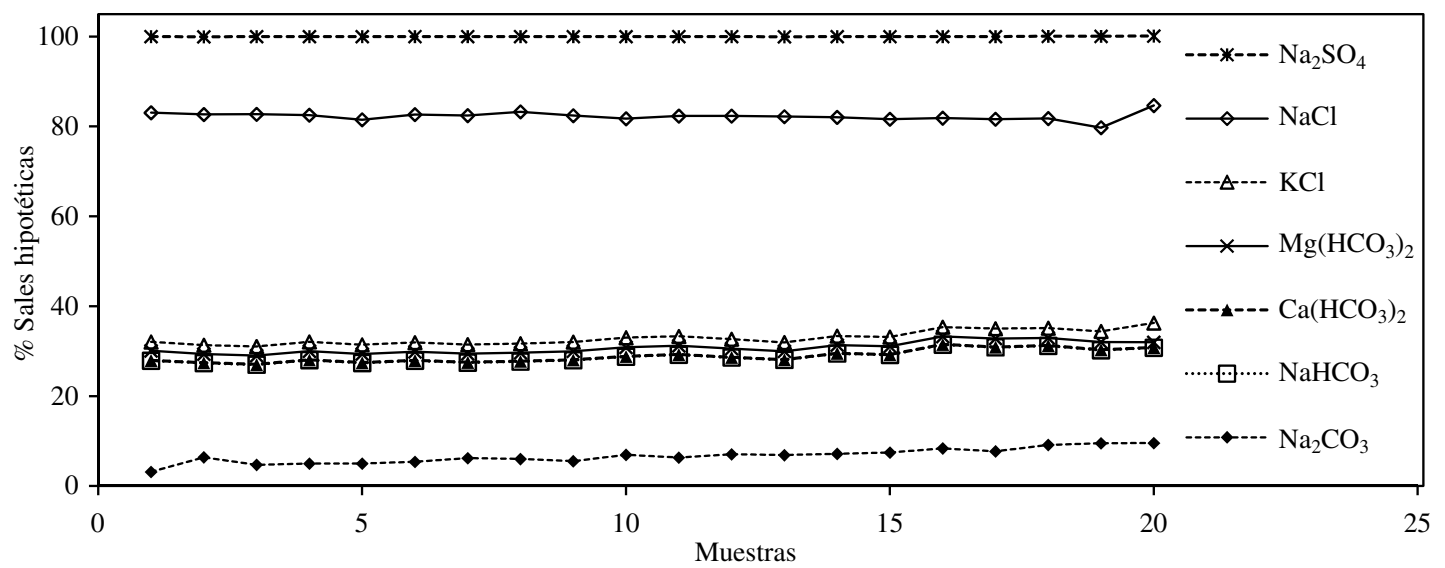

Figura 10. Sales hipotéticas, en porcentaje, de las aguas evaporadas de Zapotitlán Salinas.

En la Tabla 6 se presentan los contenidos de $\mathrm{SiO}_{2}$ en las aguas de Alchichica, Atexcac y el manantial de Zapotitlán Salinas, Puebla. En la Figura 12 se presenta la dependencia del contenido de $\mathrm{SiO}_{2}$ y del $\mathrm{pH}$, en las soluciones secuenciadas (cada $100 \mathrm{ml}$ ) durante el proceso de evaporación de las aguas salinas de Alchichica, Atexcac, y las aguas del manantial de Zapotitlán, Puebla. En las soluciones salinas que han evolucionado hacia la vía alcalina carbonatada, se presentan procesos de alta alcalinidad debido a los procesos de hidrólisis continua y permanente.

Es necesario señalar que todas las aguas que circulan en la corteza terrestre poseen cantidades suficientes de moléculas orgánicas saturadas con iones de sodio. En la Figura 13 se presenta la dependencia de contenido de silicio en función del aumento de la concentración total electrolítica en las muestras de agua salina. Cabe mencionar que el contenido de silicio en las soluciones salinas corresponde a 
Tabla 5. Composición iónica de los procesos de la metamorfosis y evolución de las aguas del manantial de Zapotitlán Salinas, Puebla.

\begin{tabular}{|c|c|c|c|c|c|c|c|c|c|c|c|c|c|c|c|}
\hline \multirow{2}{*}{$\mathbf{N}^{0}$} & \multirow{2}{*}{ pH } & \multirow{2}{*}{$\begin{array}{c}\mathrm{CE} \\
\mu \mathrm{S} \mathrm{\text {cm}^{-1 }}\end{array}$} & $\mathrm{Ca}^{2+}$ & $\mathrm{Mg}^{2+}$ & $\mathrm{Na}^{+}$ & $\mathbf{K}^{+}$ & \multirow{2}{*}{ Suma } & $\mathrm{CO}_{3}{ }^{2-}$ & $\mathrm{HCO}_{3}^{-}$ & $\mathrm{Cl}^{-}$ & $\mathrm{SO}_{4}{ }^{2-}$ & \multirow{2}{*}{ Suma } & \multirow{2}{*}{$\begin{array}{c}\% \\
\text { error }\end{array}$} & \multirow{2}{*}{$\operatorname{mg~L}^{\mathrm{mmol} \mathrm{L}^{-1}}$} & \multirow{2}{*}{$\begin{array}{c}\text { RSE } \\
\text { mg L }^{-1}\end{array}$} \\
\hline & & & \multicolumn{4}{|c|}{ mmolc $L^{-1}$} & & \multicolumn{4}{|c|}{ mmolc $L^{-1}$} & & & & \\
\hline 1 & 8,3 & 27840 & 0,16 & 5,70 & 261,80 & 5,30 & 272,96 & 8,40 & 72,00 & 141,60 & 45,30 & 267,30 & 1,77 & & 18506,20 \\
\hline & & & 3,21 & 69,20 & 6020,90 & 207,60 & & 252,00 & 4392,70 & 5021,50 & 2176,20 & & & 18143,30 & \\
\hline & & & 0,08 & 2,80 & 261,80 & 5,30 & & 4,20 & 72,00 & 141,60 & 22,70 & & & 510,50 & \\
\hline 2 & 9,1 & 29920 & 0,16 & 5,50 & 280,90 & 5,90 & 292,46 & 18,40 & 66,00 & 153,30 & 49,80 & 287,50 & 1,44 & & 19360,60 \\
\hline & & & 3,21 & 67,40 & 6461,20 & 229,10 & & 552,00 & 4026,70 & 5437,40 & 2391,90 & & & 19168,90 & \\
\hline & & & 0,08 & 2,80 & 280,90 & 5,90 & & 9,20 & 66,00 & 153,30 & 24,90 & & & 543,10 & \\
\hline 3 & 8,9 & 30860 & 0,16 & 5,80 & 290,80 & 6,10 & 302,86 & 14,00 & 72,00 & 159,30 & 51,20 & 296,50 & 1,77 & & 20517,50 \\
\hline & & & 3,21 & 69,90 & 6687,50 & 238,10 & & 420,00 & 4392,70 & 5647,40 & 2461,10 & & & 19919,90 & \\
\hline & & & 0,08 & 2,90 & 290,80 & 6,10 & & 0,10 & 2,90 & 290,80 & 6,10 & & & 599,80 & \\
\hline 4 & 8,9 & 31600 & 0,12 & 5,90 & 297,70 & 6,40 & 310,12 & 15,20 & 76,00 & 159,70 & 53,10 & 304,00 & 1,68 & & 20884,40 \\
\hline & & & 2,40 & 71,40 & 6847,80 & 248,30 & & 456,00 & 4636,80 & 5662,30 & 2549,90 & & & 20474,90 & \\
\hline & & & 0,06 & 2,90 & 297,70 & 6,40 & & 7,60 & 76,00 & 159,70 & 26,50 & & & 576,90 & \\
\hline 5 & 8,9 & 33310 & 0,20 & 6,20 & 314,00 & 6,60 & 327,00 & 16,00 & 78,00 & 166,80 & 59,20 & 320,00 & 1,80 & & 21771,50 \\
\hline & & & 4,01 & 74,80 & 7222,70 & 257,30 & & 480,00 & 4758,80 & 5914,40 & 2843,90 & & & 21555,90 & \\
\hline & & & 0,10 & 3,10 & 314,00 & 6,60 & & 8,00 & 78,00 & 166,80 & 29,60 & & & 606,20 & \\
\hline 6 & 9,0 & 34100 & 0,16 & 6,30 & 320,60 & 6,70 & 333,76 & 17,60 & 80,00 & 172,40 & 56,70 & 326,70 & 1,82 & & 22618,20 \\
\hline & & & 3,21 & 76,40 & 7374,50 & 262,00 & & 528,00 & 4880,80 & 6112,60 & 2721,90 & & & 21959,40 & \\
\hline & & & 0,08 & 3,10 & 320,60 & 6,70 & & 8,80 & 80,00 & 172,40 & 28,30 & & & 620,00 & \\
\hline 7 & 8,9 & 34890 & 0,20 & 6,40 & 329,10 & 6,90 & 342,60 & 20,80 & 78,00 & 177,90 & 59,00 & 335,70 & 1,72 & & 22891,50 \\
\hline & & & 4,01 & 77,50 & 7569,80 & 268,60 & & 624,00 & 4758,80 & 6306,60 & 2833,30 & & & 22442,60 & \\
\hline & & & 0,10 & 3,20 & 329,10 & 6,90 & & 10,40 & 78,00 & 177,90 & 29,50 & & & 635,10 & \\
\hline 8 & 8,9 & 35270 & 0,20 & 6,40 & 331,80 & 6,90 & 345,30 & 20,40 & 80,00 & 181,50 & 56,70 & 338,60 & 1,67 & & 22862,60 \\
\hline & & & 4,01 & 77,30 & 7632,10 & 271,00 & & 612,00 & 4880,80 & 6437,10 & 2721,90 & & & 22636,20 & \\
\hline & & & 0,10 & 3,20 & 331,80 & 6,90 & & 10,20 & 80,00 & 181,50 & 28,30 & & & 642,00 & \\
\hline 9 & 8,9 & 36650 & 0,20 & 6,80 & 344,70 & 7,30 & 359,00 & 19,40 & 86,00 & 184,30 & 61,90 & 351,60 & 1,75 & & 24343,60 \\
\hline & & & 4,01 & 82,10 & 7928,30 & 284,60 & & 582,00 & 5246,90 & 6536,00 & 2970,70 & & & 23634,60 & \\
\hline & & & 0,10 & 3,40 & 344,70 & 7,30 & & 9,70 & 86,00 & 184,30 & 30,90 & & & 666,40 & \\
\hline 10 & 9,1 & 36600 & 0,16 & 7,10 & 343,60 & 7,60 & 358,46 & 24,40 & 84,00 & 178,70 & 64,20 & 351,30 & 1,68 & & 24038,50 \\
\hline & & & 3,21 & 86,50 & 7903,30 & 295,60 & & 732,00 & 5124,80 & 6336,30 & 3085,40 & & & 23567,10 & \\
\hline & & & 0,08 & 3,60 & 343,60 & 7,60 & & 12,20 & 84,00 & 178,70 & 32,10 & & & 661,90 & \\
\hline 11 & 9,1 & 40100 & 0,18 & 7,50 & 377,30 & 8,10 & 393,08 & 34,40 & 96,00 & 197,20 & 68,20 & 395,80 & 0,60 & & 26506,70 \\
\hline & & & 3,61 & 91,00 & 8677,70 & 315,50 & & 1032,00 & 5857,00 & 6991,30 & 3276,10 & & & 26244,20 & \\
\hline & & & 0,09 & 3,70 & 377,30 & 8,10 & & 17,20 & 96,00 & 197,20 & 34,10 & & & 733,70 & \\
\hline 12 & 9,1 & 42500 & 0,18 & 7,90 & 380,10 & 8,60 & 396,78 & 28,80 & 96,00 & 197,20 & 68,20 & 390,20 & 1,41 & & 26950,40 \\
\hline & & & 3,61 & 95,50 & 8742,80 & 335,10 & & 864,00 & 5857,00 & 6991,30 & 3276,10 & & & 26165,40 & \\
\hline & & & 0,09 & 3,90 & 380,10 & 8,60 & & 14,40 & 96,00 & 197,20 & 34,10 & & & 734,40 & \\
\hline 13 & 9,1 & 44200 & 0,16 & 7,80 & 417,00 & 8,60 & 433,56 & 29,20 & 98,00 & 221,80 & 75,40 & 424,40 & 1,79 & & 28930,80 \\
\hline & & & 3,21 & 95,30 & 9589,90 & 334,30 & & 876,00 & 5979,00 & 7864,30 & 3621,50 & & & 28363,50 & \\
\hline & & & 0,08 & 3,90 & 417,00 & 8,60 & & 14,60 & 98,00 & 221,80 & 37,70 & & & 801,70 & \\
\hline 14 & 9,2 & 46400 & 0,16 & 8,10 & 438,00 & 9,00 & 455,26 & 32,00 & 108,00 & 226,20 & 80,20 & 446,40 & 1,65 & & 30248,10 \\
\hline & & & 3,21 & 98,50 & 10073,10 & 352,70 & & 960,00 & 6589,10 & 8021,40 & 3850,60 & & & 29948,60 & \\
\hline & & & 0,08 & 4,10 & 438,00 & 9,00 & & 16,00 & 108,00 & 226,20 & 40,10 & & & 841,50 & \\
\hline 15 & 9,2 & 52000 & 0,14 & 9,20 & 490,70 & 10,50 & 510,54 & 37,20 & 118,00 & 252,50 & 91,80 & 499,50 & 1,82 & & 34495,60 \\
\hline & & & 2,81 & 112,10 & 11285,90 & 410,20 & & 1116,00 & 7199,20 & 8955,10 & 4409,60 & & & 33490,90 & \\
\hline & & & 0,07 & 4,60 & 490,70 & 10,50 & & 18,60 & 118,00 & 252,50 & 45,90 & & & 940,90 & \\
\hline 16 & 9,2 & 55200 & 0,16 & 9,40 & 520,60 & 11,10 & 541,26 & 44,40 & 132,00 & 257,50 & 96,10 & 530,00 & 1,75 & & 36367,70 \\
\hline & & & 3,21 & 114,10 & 11972,70 & 432,10 & & 1332,00 & 8053,30 & 9132,00 & 4615,20 & & & 35654,60 & \\
\hline & & & 0,08 & 4,70 & 520,60 & 11,10 & & 22,20 & 132,00 & 257,50 & 48,00 & & & 996,20 & \\
\hline 17 & 9,2 & 58800 & 0,16 & 10,80 & 553,30 & 12,50 & 576,76 & 43,60 & 141,70 & 275,60 & 103,70 & 564,60 & 1,78 & & 38432,40 \\
\hline & & & 3,21 & 130,70 & 12726,40 & 487,20 & & 1308,00 & 8643,30 & 9771,40 & 4981,70 & & & 38051,90 & \\
\hline & & & 0,08 & 5,40 & 553,30 & 12,50 & & 21,80 & 141,70 & 275,60 & 51,90 & & & 1062,30 & \\
\hline 18 & 9,3 & 70400 & 0,04 & 11,40 & 665,90 & 14,90 & 692,24 & 62,00 & 160,80 & 330,70 & 123,80 & 677,30 & 1,84 & & 46739,60 \\
\hline & & & 0,80 & 138,90 & 15315,90 & 581,80 & & 1860,00 & 9809,20 & 11725,60 & 5946,10 & & & 45378,30 & \\
\hline & & & 0,02 & 5,70 & 665,90 & 14,90 & & 31,00 & 160,80 & 330,70 & 61,90 & & & 1270,90 & \\
\hline 19 & 9,3 & 72700 & 0,06 & 11,90 & 684,30 & 16,70 & 712,96 & 66,40 & 156,50 & 333,00 & 141,90 & 697,80 & 1,78 & & 47630,60 \\
\hline & & & 1,20 & 144,90 & 15739,40 & 651,40 & & 1992,00 & 9546,20 & 11807,10 & 6814,50 & & & 46696,70 & \\
\hline & & & 0,03 & 6,00 & 684,30 & 16,70 & & 33,20 & 156,50 & 333,00 & 70,90 & & & 1300,60 & \\
\hline 20 & 9,2 & 112000 & 0,06 & 12,20 & 1042,50 & 46,40 & 1101,16 & 102,30 & 241,00 & 567,70 & 166,60 & 1077,60 & 1,86 & & 72556,10 \\
\hline & & & 1,20 & 148,00 & 23976,40 & 1814,20 & & 3067,80 & 14701,00 & 20129,20 & 7999,90 & & & 71837,70 & \\
\hline & & & 0,03 & 6,10 & 1042,50 & 46,40 & & 51,10 & 241,00 & 567,70 & 83,30 & & & 2038,10 & \\
\hline
\end{tabular}




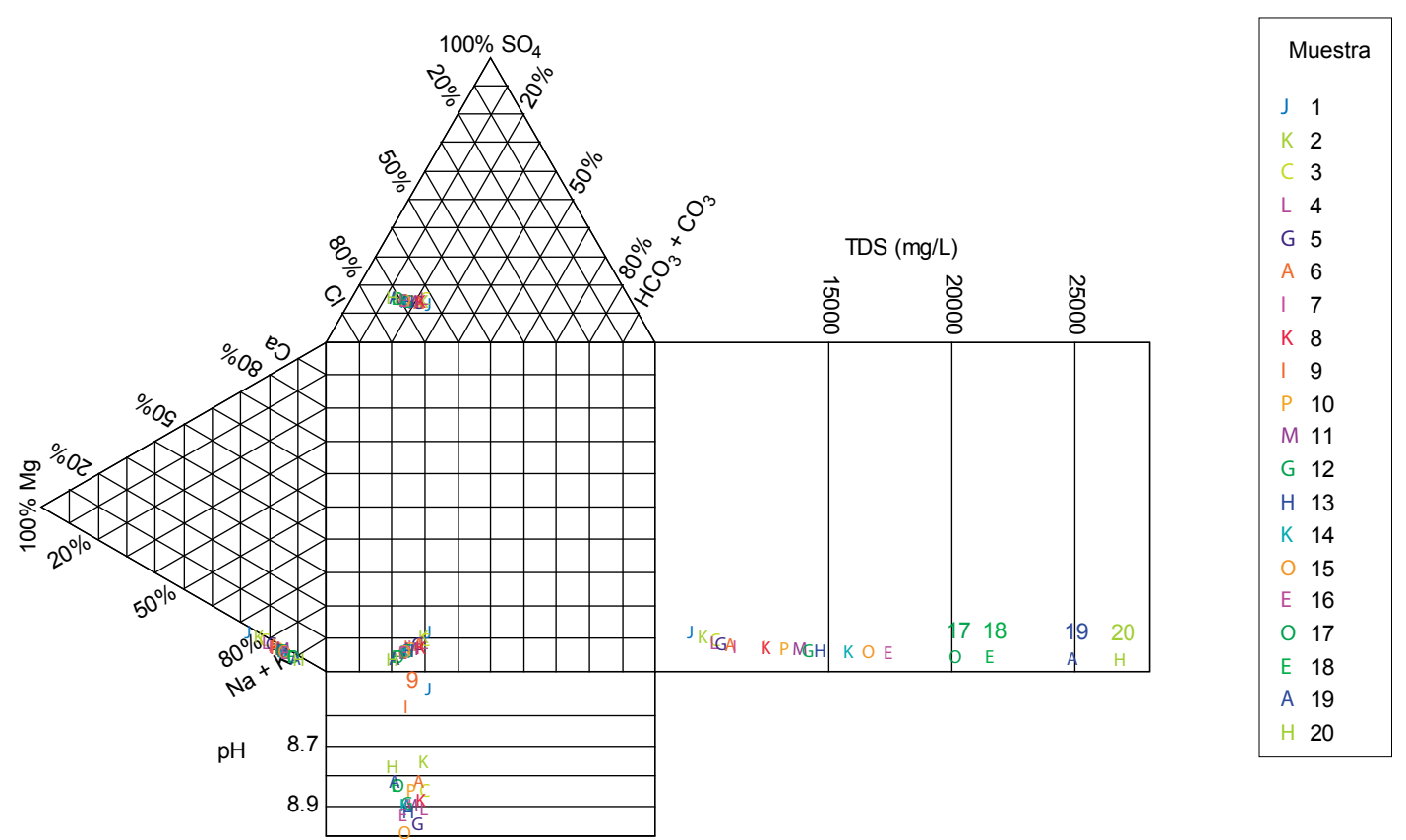

Figura 11. Diagrama de Durov, para las aguas de Zapotitlán Salinas.

Tabla 6. Concentración de silicio para las aguas del proceso de evaporación y evolución de las aguas de Alchichica, Atexcac y del manantial de Zapotitlán Salinas, Puebla.

\begin{tabular}{rrrrrr}
\hline \multicolumn{2}{c}{ ALCHICHICA } & \multicolumn{2}{c}{ ATEXCAC } & \multicolumn{2}{c}{ ZAPOTITLÁN SALINAS } \\
\hline Muestra & $\mathrm{SiO}_{2}(\mathrm{ppm})$ & Muestra & $\mathrm{SiO}_{2}(\mathrm{ppm})$ & Muestra $_{2}(\mathrm{ppm})$ \\
\hline $\mathbf{1}$ & 1,59 & 1 & 32,68 & 1 & 8,49 \\
$\mathbf{2}$ & 1,12 & 2 & 30,23 & 2 & 8,96 \\
$\mathbf{3}$ & 0,98 & 3 & 28,85 & 3 & 8,76 \\
$\mathbf{4}$ & 0,49 & 4 & 29,09 & 4 & 10,48 \\
$\mathbf{5}$ & 0,33 & 5 & 29,72 & 5 & 11,29 \\
$\mathbf{6}$ & 0,35 & 6 & 29,37 & 6 & 10,83 \\
$\mathbf{7}$ & 0,39 & 7 & 30,49 & 7 & 12,36 \\
$\mathbf{8}$ & 0,73 & 8 & 29,86 & 9 & 15,53 \\
$\mathbf{9}$ & 1,51 & 9 & 30,25 & 10 & 15,81 \\
$\mathbf{1 0}$ & 0,84 & 10 & 30,60 & 11 & 19,37 \\
$\mathbf{1 1}$ & 1,01 & 11 & 32,35 & 12 & 21,69 \\
$\mathbf{1 2}$ & 0,82 & 12 & 31,91 & 13 & 22,65 \\
$\mathbf{1 3}$ & 1,30 & 13 & 32,94 & 14 & 24,92 \\
$\mathbf{1 4}$ & 1,29 & 14 & 33,58 & 15 & 29,24 \\
$\mathbf{1 5}$ & 1,12 & 15 & 36,12 & 16 & 29,08 \\
$\mathbf{1 6}$ & 1,18 & 16 & 36,82 & 17 & 31,56 \\
$\mathbf{1 7}$ & 2,39 & 17 & 39,32 & 18 & 33,45 \\
$\mathbf{1 8}$ & 2,12 & 18 & 43,50 & 20 & 33,67 \\
$\mathbf{1 9}$ & 2,89 & 19 & 44,37 & 51,51 & 59 \\
$\mathbf{2 0}$ & 3,18 & 20 & & & \\
\end{tabular}




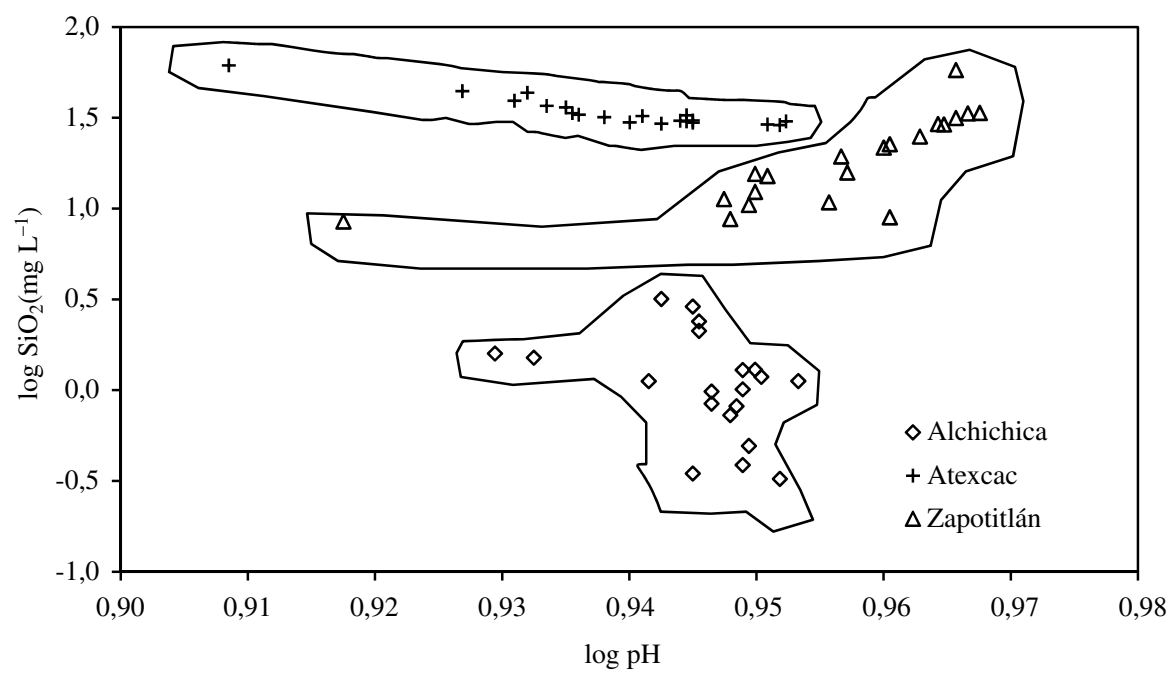

Figura 12. Contenido de silicio $\left(\mathrm{SiO}_{2}\right)$ versus $\mathrm{pH}$ en las aguas salinas de los lagos-cráter de Alchichica y Atexcac, y el manantial de Zapotitlán, durante los procesos de evaporación sucesiva.

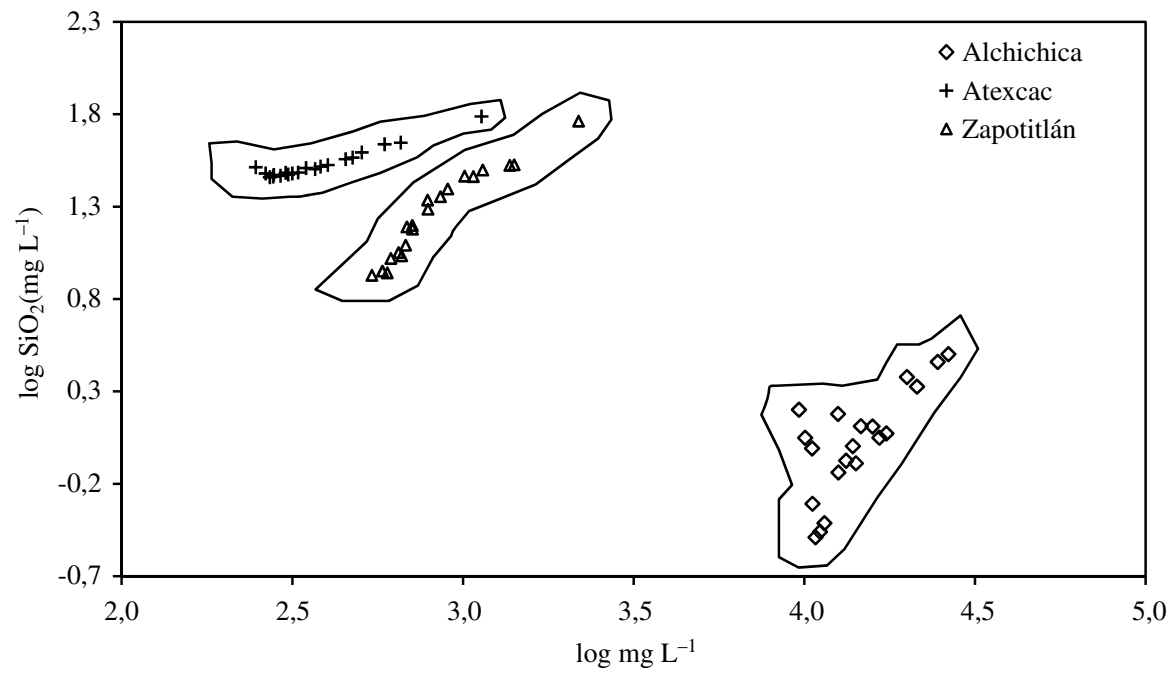

Figura 13. Contenido de silicio $\left(\mathrm{SiO}_{2}\right)$ versus $\mathrm{mg} \mathrm{L}^{-1}$ totales de las soluciones salinas secuenciadas cada $50 \mathrm{ml}$ durante un proceso de evaporación de las aguas de Alchichica, Atexcac y Zapotitlán, Puebla.

aquel que se mantiene en solución. Las reacciones alcalinas que mantienen una alta alcalinidad en las aguas salinas alcalinas se enlistan enseguida (Kovda et al., 1967). Hum-Na $+\mathrm{HOH} \rightleftarrows \mathrm{Hum}$ $\mathrm{H}+\mathrm{NaOH} ; \mathrm{Na}_{2} \mathrm{SiO}_{3}+2 \mathrm{HOH} \rightleftarrows\left(\mathrm{SiO}_{2}\right) \cdot \mathrm{H}_{2} \mathrm{O}+$ $\underline{2 \mathrm{NaOH}} ; 2 \mathrm{NaAl}_{2}+2 \mathrm{HOH} \rightleftarrows \mathrm{Al}(\mathrm{OH})_{3}+\underline{\mathrm{NaOH}}$

De acuerdo con Badaut y Risacher (1983), es necesario resaltar que la reacción que mantiene al $\mathrm{SiO}_{2}$ en solución alcalina es la siguiente: $\mathrm{Na}_{2} \mathrm{SiO}_{3}$ $+2 \mathrm{HOH} \rightleftarrows\left(\mathrm{SiO}_{2}\right) \cdot \mathrm{H}_{2} \mathrm{O}+2 \mathrm{NaOH}$. Del análisis del contenido del $\mathrm{SiO}_{2}$ en función del pH en las soluciones salinas de las diferentes fuentes de agua, se puede concluir que el contenido de $\mathrm{SiO}_{2}$ en los lagos-cráter son concentraciones que tienen su origen debido a procesos de hidrotermalismo residual que tiene lugar a partir de las explosiones volcánicas cuando se formaron estos cráteres (Yáñez y García, 1982).

En el caso particular de las aguas salinas del manantial de Zapotitlán, se debe mencionar que el sinnúmero de surgencias que se presenta en la zona de Zapotitlán se debe a evaporitas marinas sepultadas 
y a sedimentos salinos carbonatados que tuvieron su origen durante las explosiones volcánicas que levantaron la zona de Zapotitlán durante el Terciario (Reyes, 1998). Adicionalmente se debe mencionar que las aguas salinas de Zapotitlán deben su origen al paso de aguas pluviales a través de las rocas calizas sedimentarias de Zapotitlán.

\section{Conclusiones}

Las aguas superficiales de baja concentración de los estados de Tlaxcala, Puebla y Veracruz poseen cantidades prevalecientes de bicarbonato. $\mathrm{La}$ concentración de $\mathrm{HCO}_{3}$ es de 1,2 a 6,4 $\mathrm{mmol}_{\mathrm{c}} \mathrm{L}^{-1}$.

Las aguas de Tlaxcala y parcialmente de Puebla presentan concentraciones bajas y moderadas de bicarbonatos, que se deben al intemperismo de rocas sedimentarias, lutitas, tobas y espesores basálticos y riolíticos de regiones del eje neovolcánico.

Las concentraciones relativas y prevalecientes de iones de calcio y de bicarbonato en las aguas de Veracruz se deben al intemperismo de calizas. Las concentraciones de calcio son de 1,8 a 5,3 $\mathrm{mmol}_{\mathrm{c}} \mathrm{L}_{-1}$.

Las aguas altamente salinas de los lagos-cráter de Alchichica, Atexcac y del manantial de Salinas Chiquitas de Zapotitlán, Puebla, son altamente sódicas. Las sales que prevalecen son: cloruro de sodio $(\mathrm{NaCl})$, sulfato de sodio $\left(\mathrm{Na}_{2} \mathrm{SO}_{4}\right)$ y carbonato de sodio $\left(\mathrm{Na}_{2} \mathrm{CO}_{3}\right)$.

La gráfica geoquímica $\operatorname{logNa}-\log \mathrm{Cl}$ (mmolc $\mathrm{L}^{-1}$ ), de muestras de agua de variadas composiciones, permite delimitar aguas bicarbonatadas de baja concentración de aquellas aguas de alta concentración ricas en cloruros.

En las aguas de los lagos-cráter de Alchichica, Atexcac y del manantial de Salinas Chiquitas de Zapotitlán, los contenidos de silicio $\left(\mathrm{SiO}_{2}\right)$ después de un proceso de evaporación, son altos: Alchichica 0,32-3,2 mg L${ }^{-1}$; Atexcac 25,11-63,0 $\mathrm{mg} \mathrm{L}^{-1}$; Zapotitlán 6,3-60 mg L ${ }^{-1}$. Estos valores de $\mathrm{SiO}_{2}$ se deben a pH altos 9-10.

La vía geoquímica que siguen las aguas de baja concentración es la vía alcalina bicarbonatada, mientras que las aguas de alta concentración de Alchichica, Atexcac y Zapotitlán toman la vía sulfatada alcalina.

Cuando se trate de estudios de caracterización fisicoquímica de un gran conjunto de aguas superficiales, es necesario clasificar a las diferentes aguas desde el punto de vista geoquímico. Los parámetros que deben considerarse son: $\log \mathrm{Ca}$ $\log \mathrm{Alc} ; \log \mathrm{Ca}+\mathrm{Mg}-\log \mathrm{Alc} ; \log \mathrm{Ca}-\log \mathrm{Alc}_{-} \mathrm{SO}_{4} \mathrm{y}$ $\log \mathrm{Na}-\log \mathrm{Cl}$.

\section{Literatura Citada}

Alcocer, D.J.; Escolero, F.Ó.A. y Marín, S.L.E.

2005. Problemática del agua de la Cuenca Oriental, estados de Puebla, Veracruz y Tlaxcala.. En: B. Jiménez y L. Marín (eds.). El agua en México vista desde la academia. Academia Mexicana de Ciencias. México, D.F., pp. 57-77.

American Public Health Association (APHA)

1995. Standard methods for examination of water and wastewater. APHA (American Public Health Association), WWA (American Water Works Association), WPCF (Water Pollution Control Federation), Washington D.C., USA. $1035 \mathrm{pp}$.

Arredondo, F.J.; Borrego, R.L.C.; Valladolid, M. 1983. Batimetría y morfometría de los lagos "maars" de la Cuenca de Oriental, Puebla, México. Biótica, 8 (1): 31-47.

Arredondo-Figueroa, J.L.

2002. Los axalapascos de la Cuenca Oriental, Puebla. En: De la Lanza EG y García CJL (2002) (compiladores). Lagos y presas de México. AGT. México, D.F., pp. 81-107.

Badaut, D.; Risacher, F.

1983. Authigenic smectite on diatom frustules in Bolivian saline lakes. Geochim. Cosmochim. Acta, 47: 363-375.

Can, C.A.

2011 Origen y calidad del agua subterránea en la cuenca del Oriental, Puebla. Terra Latinoamericana 29: 189-200.
Durov, S.A.

1948. Natural waters and Graphic representation of their composition. Dokl. Akad. Nauk SSSR, 59: 87-90.

Eaton, A.D.; Clesceri, L.S.; Greenberg, A.E.

1995. Standard methods for the examination of water and wastewater. 19th Edition. APHA, AWWA, WEF. Washington, USA. 1325 pp.

Eugster, H.P.; Hardie, L.A.

1978 Saline lakes. En: Lakes, chemistry, geology, physics, (A. Lerman, ed.), Springer-Verlag. 237-293.

Gasca-Durán, A.

1982. Génesis de los lagos cráter de la Cuenca de Oriental. Colec. Cient. Inst. Nal. Antropol. Hist. 98 México. 45 pp.

Hardie, L.A.; Eugster, H.P.

1970. The evolution of closed-basin brines. Mineral Soc. Amer. Spec. Paper, 3: 273-290.

Kovda, V.A.; Vanden-Berg, C.C.; Hogan, R.M.

1967. International source book on Irrigation and drainage of arid lands in relation to salinity and alkalinity. FAO/ UNESCO. 267 pp.

MacNeish, R.S.; Fowler, M.L.; Cook, A.G.; Peterson, F.A.; Nelken-Terner, A.; Neely, J.

1972. The Prehistory of the Tehuacan Valley. Vol. 5: Excavations and Reconnaissance. University of Texas Press, 341-495. 


\section{Reyes-Cortés, M.}

1979. Geología de la Cuenca de Oriental, estados de Puebla, Veracruz y Tlaxcala. SEP-INAH, Colección Científica, Prehistoria 71. México, D.F. 62 pp.

Richards, L.A.

1990. Diagnóstico y rehabilitación de suelos salinos y sódicos: Manual No. 60. Sexta reimpresión. Departamento de Agricultura de los EE.UU., Laboratorio de Salinidad. Limusa. México. 172 pp.

Risacher, F.; Alonso, H.

1996a. Geoquímica del salar de Atacama, parte 1: Origen de los componentes y balance salino. Revista Geológica de Chile, 23 (1): 113-122.

Risacher, F.; Alonso, H.

1996b. Geoquímica del salar de Atacama, parte 2: Evolución de las aguas. Revista Geológica de Chile, 23 (1): 123-134.
Risacher, F.; Alonso, H.; Salazar, C.

1999 Geoquímica de aguas en cuencas cerradas I, II y III regiones-Chile. Convenio de cooperación DGA-UCNORSTOM (Síntesis). 89 pp.

Risacher, F.; Fritz, B.

1991. Geochemistry of Bolivian salars, Lipez, southern Altiplano: origin of solutes and brine evolution. Geochim. Cosmochim. Acta, 55: 687-705.

Yáñez-García, C.; García, D.S.

1982. Exploración de la región geotérmica Los HumerosLas Derrumbadas, Estados de Puebla y Veracruz. C.F.E. $96 \mathrm{pp}$.

Zaporozec, A.

1972. Graphical interpretation of water-quality data. Ground Water, 10(2): 32-43. 
